MPP-2005-28

hep-ph/0509084

\title{
Electroweak higher-order effects and theoretical uncertainties in deep-inelastic neutrino scattering
}

\author{
K.-P. O. Diener ${ }^{1}$, S. DitTmaier ${ }^{2}$ and W. Hollik ${ }^{2}$ \\ ${ }^{1}$ Paul-Scherrer-Institut, Würenlingen und Villigen \\ CH-5232 Villigen PSI, Switzerland \\ ${ }^{2}$ Max-Planck-Institut für Physik (Werner-Heisenberg-Institut) \\ D-80805 München, Germany
}

\begin{abstract}
:
A previous calculation of electroweak $\mathcal{O}(\alpha)$ corrections to deep-inelastic neutrino scattering, as e.g. measured by $\mathrm{NuTeV}$ and NOMAD, is supplemented by higher-order effects. In detail, we take into account universal two-loop effects from $\Delta \alpha$ and $\Delta \rho$ as well as higherorder final-state photon radiation off muons in the structure function approach. Moreover, we make use of the recently released $\mathcal{O}(\alpha)$-improved parton distributions MRST2004QED and identify the relevant QED factorization scheme, which is DIS like. As a technical byproduct, we describe slicing and subtraction techniques for an efficient calculation of a new type of real corrections that are induced by the generated photon distribution. A numerical discussion of the higher-order effects suggests that the remaining theoretical uncertainty from unknown electroweak corrections is dominated by non-universal two-loop effects and is of the order 0.0003 when translated into a shift in $\sin ^{2} \theta_{\mathrm{W}}=1-M_{\mathrm{W}}^{2} / M_{\mathrm{Z}}^{2}$. The $\mathcal{O}(\alpha)$ corrections implicitly included in the parton distributions lead to a shift of about 0.0004 .
\end{abstract}

September 2005 


\section{Introduction}

In 2002 the $\mathrm{NuTeV}$ collaboration has deduced the on-shell weak mixing angle, $\sin ^{2} \theta_{\mathrm{W}}=1-M_{\mathrm{W}}^{2} / M_{\mathrm{Z}}^{2}$, from the ratio of neutral- to charged-current cross-sections measured in deep-inelastic neutrino scattering [1]. The NuTeV result on $\sin ^{2} \theta_{\mathrm{W}}$ is about $3 \sigma$ at variance with the overall fit [2] of the Standard Model (SM) to precision observables, a fact that is known as "the NuTeV anomaly". In spite of many attempts to understand or to resolve this anomaly in or beyond [3-7] the SM, the issue is still not settled. Inside the SM, uncertainties originating from parton distributions [4,5,7-10] (isospin violation, strange-sea asymmetry), from nuclear effects $[4,7,9,11]$, and from QCD $[3,9,12]$ and electroweak [13-16] radiative corrections significantly influence the $\mathrm{NuTeV}$ result. In view of the recent progress on these subjects, a careful reanalysis of the $\mathrm{NuTeV}$ result, including its error assessment, would be welcome. In this context it is very interesting that the NOMAD collaboration plans to extract $\sin ^{2} \theta_{\mathrm{W}}$ from their data on neutrino scattering [17].

In our previous work [15], we performed a calculation of the electroweak corrections of $\mathcal{O}(\alpha)$ to neutral- and charged-current deep-inelastic neutrino scattering and compared our results with the older calculation [14] which was used in the $\mathrm{NuTeV}$ data analysis. Unfortunately, we could not fully reproduce the results of Ref. [14], which was in part certainly due to the incompletely known setup and input. However, a comparison with a more recent recalculation [16], which is in line with Ref. [14], suggests that the still remaining differences are due to different kinematical approximations made in Refs. $[14,16]$ compared to our calculation. While our calculation [15] is a pure $\mathcal{O}(\alpha)$ calculation without further approximations, in Refs. [14,16] the momentum transfer of the $\mathrm{W}$ and $\mathrm{Z}$ bosons was neglected, i.e. the approximation of a four-fermion contact interaction was made. Moreover, we concluded in Ref. [15] that the estimate of remaining theoretical uncertainties from unknown electroweak higher orders was too optimistic.

In this paper, we refine our previous calculation [15] of the electroweak corrections to deep-inelastic neutrino scattering by including leading effects beyond $\mathcal{O}(\alpha)$, in order to further reduce the theoretical uncertainty in the electroweak sector. In detail, after a short summary of our conventions in Section 2 we work out the leading universal corrections originating from the running of the electromagnetic coupling and from the $\rho$ parameter in various input-parameter schemes in Section 3, in order to reduce the scheme dependence. Moreover, in Section 4 we include the leading logarithmic effects beyond $\mathcal{O}(\alpha)$ that result from collinear final-state radiation (FSR) in the structure-function approach. Finally, we make use of the recently released $\mathcal{O}(\alpha)$-improved parton distribution functions (PDFs) of the MRST collaboration [10], which requires two kind of refinements, as explained in Section 5.1. Firstly, the factorization scheme for the $\mathcal{O}(\alpha)$ corrections has to be chosen in a way that is consistent with the treatment of $\mathcal{O}(\alpha)$ corrections used in the fit of the PDFs. Secondly, the $\mathcal{O}(\alpha)$ driven evolution of the PDFs leads to an induced photon distribution, so that a new type of real corrections with a photon in the initial state has to be considered. For a proper technical treatment of the photon-induced processes we have worked out different variants of phase-space slicing and dipole subtraction, as described in the Appendix in detail. Section 6 contains our numerical results, which comprise a discussion of the various higher-order corrections both to integrated and differential cross 
sections. Moreover, we derive an estimate of the remaining theoretical uncertainties from still missing electroweak effects there. Our conclusions are given in Section 7.

\section{Kinematics and conventions}

In this section we briefly repeat our conventions of Ref. [15] as far as relevant in the sequel. We consider the neutral-current (NC) and charged-current (CC) parton processes

$$
\begin{array}{ll}
\mathrm{NC}: & \nu_{\mu}\left(p_{l}\right)+q\left(p_{q}\right) \rightarrow \nu_{\mu}\left(k_{l}\right)+q\left(k_{q}\right), \quad q=\mathrm{u}, \mathrm{d}, \mathrm{s}, \mathrm{c}, \overline{\mathrm{u}}, \overline{\mathrm{d}}, \overline{\mathrm{s}}, \overline{\mathrm{c}}, \\
\mathrm{CC}: & \nu_{\mu}\left(p_{l}\right)+q\left(p_{q}\right) \rightarrow \mu^{-}\left(k_{l}\right)+q^{\prime}\left(k_{q}\right), \quad q=\mathrm{d}, \mathrm{s}, \overline{\mathrm{u}}, \overline{\mathrm{c}}, \quad q^{\prime}=\mathrm{u}, \mathrm{c}, \overline{\mathrm{d}}, \overline{\mathrm{s}},
\end{array}
$$

and the processes with all particles replaced by their antiparticles. The assignment of momenta is indicated in parentheses. At lowest order the former processes proceed via Z-boson exchange, the latter via W-boson exchange. CP symmetry implies that the (parton) cross sections for $\nu_{\mu}$-quark and $\nu_{\mu}$-antiquark scattering are equal to the ones of $\bar{\nu}_{\mu}$-antiquark and $\bar{\nu}_{\mu}$-quark scattering, respectively.

In the discussion of numerical results, we concentrate on the $\mathrm{NC}$ to $\mathrm{CC}$ cross-section ratio [18]

$$
R^{\nu}=\frac{\sigma_{\mathrm{NC}}^{\nu}\left(\nu_{\mu} N \rightarrow \nu_{\mu} X\right)}{\sigma_{\mathrm{CC}}^{\nu}\left(\nu_{\mu} N \rightarrow \mu^{-} X\right)},
$$

but our calculation is applicable to any combination of $\mathrm{NC}$ and $\mathrm{CC}$ cross section involving incoming neutrinos or anti-neutrinos, such as the ratio

$$
R^{-}=\frac{\sigma_{\mathrm{NC}}^{\nu}\left(\nu_{\mu} N \rightarrow \nu_{\mu} X\right)-\sigma_{\mathrm{NC}}^{\bar{\nu}}\left(\bar{\nu}_{\mu} N \rightarrow \bar{\nu}_{\mu} X\right)}{\sigma_{\mathrm{CC}}^{\nu}\left(\nu_{\mu} N \rightarrow \mu^{-} X\right)-\sigma_{\mathrm{CC}}^{\bar{\nu}}\left(\bar{\nu}_{\mu} N \rightarrow \mu^{+} X\right)},
$$

as proposed by Paschos and Wolfenstein [19].

Throughout the calculation, we neglect the muon mass and the parton masses whenever possible. The quark masses are only kept as regulators for mass singularities, which appear as mass logarithms in the (photonic) corrections; the quark-mass logarithms are absorbed into the PDFs as described in Section 5. The muon mass is not only required as regulator for collinear final-state radiation but also for a proper description of forward scattering in the (loop-induced) $\gamma \nu_{\mu} \bar{\nu}_{\mu}$ vertex, as described in Ref. [15] in detail.

The momentum of the incoming (anti-)quark $q$ is related to the total nucleon momentum $P_{N}$ by the usual scaling relation

$$
p_{q}^{\mu}=x P_{N}^{\mu}
$$

which holds in the centre-of-mass (CM) frame of the partonic system where the nucleon mass $M_{N}$ is negligible. The variable $x$ is the usual momentum fraction, restricted by $0<x<1$. Since deep-inelastic neutrino scattering usually is accessed in fixed-target experiments (as $\mathrm{NuTeV}$ ), we take the energy $E_{\nu}^{\mathrm{LAB}}$ of the incoming (anti-)neutrino beam to define the incoming momentum $p_{l}$ in the rest frame of the nucleon, called LAB in the following. Thus, the squared partonic CM energy is given by

$$
s=\left(p_{l}+p_{q}\right)^{2}=2 x M_{N} E_{\nu}^{\mathrm{LAB}},
$$


up to terms of higher order in the nucleon mass. Moreover, we define the Bjorken variable

$$
x_{\mathrm{Bj}}=\frac{-\left(p_{l}-k_{l}\right)^{2}}{2 P_{N}\left(p_{l}-k_{l}\right)}=\frac{-\left(p_{l}-k_{l}\right)^{2}}{2 M_{N} E_{\mathrm{had}+\text { phot }}^{\mathrm{LAB}}}
$$

and the energy ratio $y$ via

$$
y=\frac{E_{\mathrm{had}+\mathrm{phot}}^{\mathrm{LAB}}}{E_{\nu}^{\mathrm{LAB}}}
$$

where $E_{\text {had+phot }}^{\mathrm{LAB}}$ is the energy (in the LAB frame) deposited by hadrons and photons in the detector. In lowest order $x_{\mathrm{Bj}}$ and $x$ coincide, and $E_{\text {had }+ \text { phot }}^{\mathrm{LAB}}$ is identical with the energy of the outgoing quark; in the presence of photon (or gluon) radiation this is not the case anymore.

\section{$3 \quad$ Universal electroweak corrections beyond $\mathcal{O}(\alpha)$}

The integrated $\mathrm{CC}$ and $\mathrm{NC}$ cross sections at parton level, including $\mathcal{O}(\alpha)$ corrections, can be written as

$$
\begin{gathered}
\left.\sigma_{\mathrm{CC}, 1}\right|^{\mathrm{IPS}}=\left.\sigma_{\mathrm{CC}, 0}\right|^{\mathrm{IPS}}\left(1+\left.\delta_{\mathrm{CC}, 1}\right|^{\mathrm{IPS}}\right), \\
\left.\sigma_{\mathrm{NC}, 1}^{\tau}\right|^{\mathrm{IPS}}=\left.\sigma_{\mathrm{NC}, 0}^{\tau}\right|^{\mathrm{IPS}}\left(1+\left.\delta_{\mathrm{NC}, 1}^{\tau}\right|^{\mathrm{IPS}}\right),
\end{gathered}
$$

where $\sigma_{\mathrm{CC}, 0}$ and $\sigma_{\mathrm{NC}, 0}^{\tau}$ denote the partonic lowest-order cross sections, and $\tau= \pm$ refers to the chirality of the quark line. The input-parameter scheme (IPS) is indicated by an upper index which takes the value $\alpha(0), \alpha\left(M_{\mathrm{Z}}\right)$, or $G_{\mu}$ for the respective scheme. The precise definition of these IPS is given in Section 4.2 of Ref. [15]. Roughly speaking, the whole SM input consists of all particle masses together with the chosen $\alpha$ for the electromagnetic coupling. The $\alpha(0)$-scheme corresponds to the "complete on-shell renormalization scheme" as described, e.g., in Refs. [20,21]. The relative $\mathcal{O}(\alpha)$ corrections $\delta_{\mathrm{CC}, 1}$ and $\delta_{\mathrm{NC}, 1}^{\tau}$ are proportional to the value of $\alpha$ in the corresponding IPS, where $\alpha_{G_{\mu}}=\sqrt{2} G_{\mu} M_{\mathrm{W}}^{2} s_{\mathrm{w}}^{2} / \pi$ in the $G_{\mu}$-scheme. Apart from this factor the relative corrections differ by constant terms,

$$
\begin{aligned}
\left.\delta_{1}\right|^{\alpha\left(M_{\mathrm{Z}}\right)} & =\frac{\alpha\left(M_{\mathrm{Z}}\right)}{\alpha(0)}\left[\left.\delta_{1}\right|^{\alpha(0)}-2 \Delta \alpha\left(M_{\mathrm{Z}}\right)\right], \\
\left.\delta_{1}\right|^{G_{\mu}} & =\frac{\alpha_{G_{\mu}}}{\alpha(0)}\left[\left.\delta_{1}\right|^{\alpha(0)}-2 \Delta r_{1}\right] .
\end{aligned}
$$

Here

$$
\Delta \alpha\left(M_{\mathrm{Z}}\right)=\left.\left[\Pi^{\gamma \gamma}(0)-\operatorname{Re}\left\{\Pi\left(M_{\mathrm{Z}}^{2}\right)\right\}\right]\right|_{f \neq \mathrm{top}} \approx \frac{\alpha(0)}{3 \pi} \sum_{f \neq \mathrm{top}} N_{f}^{\mathrm{c}} Q_{f}^{2}\left[\ln \left(\frac{M_{\mathrm{Z}}^{2}}{m_{f}^{2}}\right)-\frac{5}{3}\right]
$$

describes the running of the electromagnetic coupling from the scale $Q=0$ to $Q=M_{\mathrm{Z}}$, induced by the light fermions $f$ (all but the top quark) with colour factor $N_{f}^{\mathrm{c}}$, electric charge $Q_{f}$, and mass $m_{f}$. The quantity $\Pi^{\gamma \gamma}\left(Q^{2}\right)$ is the photonic vacuum polarization in 
the convention of Ref. [20]. The quantity $\Delta r_{1}$ is the pure one-loop correction to muon decay [22], as defined by

$$
M_{\mathrm{W}}^{2}\left(1-\frac{M_{\mathrm{W}}^{2}}{M_{\mathrm{Z}}^{2}}\right)=\frac{\pi \alpha(0)}{\sqrt{2} G_{\mu}}\left[1+\Delta r_{1}\left(\alpha(0), M_{\mathrm{W}}, M_{\mathrm{Z}}, M_{\mathrm{H}}, m_{f}\right)\right] .
$$

Note that $\Delta r_{1}$ implicitly contains large contributions from $\Delta \alpha\left(M_{\mathrm{Z}}\right) \sim 6 \%$ and the (oneloop) correction $\left(c_{\mathrm{w}}^{2} / s_{\mathrm{w}}^{2}\right) \Delta \rho_{1} \sim 3 \%$ induced by the $\rho$-parameter,

$$
\Delta r_{1}=\Delta \alpha\left(M_{\mathrm{Z}}\right)-\left.\frac{c_{\mathrm{w}}^{2}}{s_{\mathrm{w}}^{2}} \Delta \rho_{1}\right|^{\alpha(0)}+\Delta r_{\mathrm{rem}}
$$

where

$$
\left.\Delta \rho_{1}\right|^{\alpha}=\frac{3 \alpha}{16 \pi s_{\mathrm{w}}^{2}} \frac{m_{\mathrm{t}}^{2}}{M_{\mathrm{W}}^{2}} .
$$

The remainder $\Delta r_{\text {rem }}$ comprises the non-universal one-loop corrections to muon decay and is of the size of $\sim 1 \%$.

The leading universal two-loop effects induced by $\Delta \alpha\left(M_{\mathrm{Z}}\right)$ and by corrections $\Delta \rho$ in the parameter $\rho=1 /(1-\Delta \rho)$ can be included as described in Ref. [23]. The procedure is as follows. Starting from lowest-order cross sections in the $\alpha(0)$-scheme, all orders $\Delta \alpha\left(M_{\mathrm{Z}}\right)^{n}$ can be effectively included upon substituting $\alpha(0) \rightarrow \alpha\left(M_{\mathrm{Z}}\right)=\alpha(0) /\left[1-\Delta \alpha\left(M_{\mathrm{Z}}\right)\right]$ as electromagnetic coupling. The effects from $\Delta \rho$ can be absorbed by replacing $s_{\mathrm{w}}^{2}$ and $c_{\mathrm{w}}^{2}$ by appropriate modifications

$$
\bar{s}_{\mathrm{w}}^{2}=s_{\mathrm{w}}^{2}+c_{\mathrm{w}}^{2} \Delta \rho, \quad \bar{c}_{\mathrm{w}}^{2}=1-\bar{s}_{\mathrm{w}}^{2} .
$$

This recipe is correct up to $\mathcal{O}\left(\Delta \rho^{2}\right)$. As shown in Ref. [23], the introduction of $\alpha\left(M_{\mathrm{Z}}\right)$ and $\bar{s}_{\mathrm{w}}^{2}, \bar{c}_{\mathrm{w}}^{2}$ also correctly reproduces the correct terms of $\mathcal{O}\left(\Delta \alpha\left(M_{\mathrm{Z}}\right) \Delta \rho\right)$ in processes with four light external fermions. Note that in $\mathcal{O}\left(\Delta \rho^{2}\right)$ both one- and two-loop corrections to $\Delta \rho$ become relevant; explicitly we use the result

$$
\Delta \rho=3 x_{\mathrm{t}}\left[1+\rho^{(2)}\left(M_{\mathrm{H}}^{2} / m_{\mathrm{t}}^{2}\right) x_{\mathrm{t}}\right]\left[1-\frac{2 \alpha_{\mathrm{s}}}{9 \pi}\left(\pi^{2}+3\right)\right], \quad 3 x_{\mathrm{t}}=\frac{3 \sqrt{2} G_{\mu} m_{\mathrm{t}}^{2}}{16 \pi^{2}}=\left.\Delta \rho_{1}\right|^{G_{\mu}}
$$

with the function $\rho^{(2)}$ given in Eq. (12) of Ref. [24]. In order to avoid double-counting, the one-loop effects of $\Delta \alpha\left(M_{\mathrm{Z}}\right)$ and $\Delta \rho$ have to be subtracted from the full one-loop corrections $\left.\delta_{1}\right|^{\alpha(0)}$ introduced above.

The above recipe can be easily modified to the other two IPS. In the $\alpha\left(M_{\mathrm{Z}}\right)$-scheme, the replacement $\alpha(0) \rightarrow \alpha\left(M_{\mathrm{Z}}\right)$ has already been done, so that the procedure reduces to the substitutions $s_{\mathrm{w}} \rightarrow \bar{s}_{\mathrm{w}}$ and $c_{\mathrm{w}} \rightarrow \bar{c}_{\mathrm{w}}$. In the $G_{\mu}$-scheme, one has to recall that $\alpha_{G_{\mu}}$ effectively involves a factor $\alpha\left(M_{\mathrm{Z}}\right) s_{\mathrm{w}}^{2}$, so that the recipe reads $\alpha_{G_{\mu}} \rightarrow \alpha_{G_{\mu}} \bar{s}_{\mathrm{w}}^{2} / s_{\mathrm{w}}^{2}, s_{\mathrm{w}} \rightarrow \bar{s}_{\mathrm{w}}$, and $c_{\mathrm{w}} \rightarrow \bar{c}_{\mathrm{w}}$; as a net effect the combination $\alpha_{G_{\mu}} / s_{\mathrm{w}}^{2}$ is not modified.

The lowest-order CC cross section is proportional to $\alpha^{2} / s_{\mathrm{w}}^{4}$. Thus, using the above recipes we find for the corrected cross sections, which include the full $\mathcal{O}(\alpha)$ corrections as well as two-loop improvements from $\Delta \alpha \equiv \Delta \alpha\left(M_{\mathrm{Z}}\right)$ and $\Delta \rho$, the results

$$
\left.\sigma_{\mathrm{CC}, 1+\mathrm{LL}^{2}}\right|^{\alpha(0)}=\left.\sigma_{\mathrm{CC}, 0}\right|^{\alpha(0)}\left[\frac{s_{\mathrm{w}}^{4}}{\bar{s}_{\mathrm{w}}^{4}} \frac{1}{(1-\Delta \alpha)^{2}}+\left.\delta_{\mathrm{CC}, 1}\right|^{\alpha(0)}-2 \Delta \alpha+\left.2 \frac{c_{\mathrm{w}}^{2}}{s_{\mathrm{w}}^{2}} \Delta \rho_{1}\right|^{\alpha(0)}\right]
$$




$$
\begin{aligned}
& =\left.\sigma_{\mathrm{CC}, 0}\right|^{\alpha(0)}\left[1+\left.\delta_{\mathrm{CC}, 1}\right|^{\alpha(0)}+2 \frac{c_{\mathrm{w}}^{2}}{s_{\mathrm{w}}^{2}}\left(\left.\Delta \rho_{1}\right|^{\alpha(0)}-\Delta \rho\right)\right. \\
& \left.+3 \Delta \alpha^{2}-4 \frac{c_{\mathrm{w}}^{2}}{s_{\mathrm{w}}^{2}} \Delta \alpha \Delta \rho+3 \frac{c_{\mathrm{w}}^{4}}{s_{\mathrm{w}}^{4}} \Delta \rho^{2}\right]+\ldots, \\
& \left.\sigma_{\mathrm{CC}, 1+\mathrm{LL}^{2}}\right|^{\alpha\left(M_{\mathrm{Z}}\right)}=\left.\sigma_{\mathrm{CC}, 0}\right|^{\alpha\left(M_{\mathrm{Z}}\right)}\left[\frac{s_{\mathrm{w}}^{4}}{\bar{s}_{\mathrm{w}}^{4}}+\left.\delta_{\mathrm{CC}, 1}\right|^{\alpha\left(M_{\mathrm{Z}}\right)}+\left.2 \frac{c_{\mathrm{w}}^{2}}{s_{\mathrm{w}}^{2}} \Delta \rho_{1}\right|^{\alpha\left(M_{\mathrm{Z}}\right)}\right] \\
& =\left.\sigma_{\mathrm{CC}, 0}\right|^{\alpha\left(M_{\mathrm{Z}}\right)}\left[1+\left.\delta_{\mathrm{CC}, 1}\right|^{\alpha\left(M_{\mathrm{Z}}\right)}+2 \frac{c_{\mathrm{w}}^{2}}{s_{\mathrm{w}}^{2}}\left(\left.\Delta \rho_{1}\right|^{\alpha\left(M_{\mathrm{Z}}\right)}-\Delta \rho\right)+3 \frac{c_{\mathrm{w}}^{4}}{s_{\mathrm{w}}^{4}} \Delta \rho^{2}\right] \\
& +\ldots, \\
& \left.\sigma_{\mathrm{CC}, 1+\mathrm{LL}^{2}}\right|^{G_{\mu}}=\left.\sigma_{\mathrm{CC}, 0}\right|^{G_{\mu}}\left[1+\left.\delta_{\mathrm{CC}, 1}\right|^{G_{\mu}}\right]=\left.\sigma_{\mathrm{CC}, 1}\right|^{G_{\mu}} .
\end{aligned}
$$

For the $\alpha(0)$ - and $\alpha\left(M_{\mathrm{Z}}\right)$-schemes the two given expressions are equal up to non-leading two-loop and leading three-loop terms. Equation (3.11) reflects the well-known fact that the $G_{\mu}$-scheme, which employs $G_{\mu}, M_{\mathrm{W}}$, and $M_{\mathrm{Z}}$ as independent parameters, effectively absorbs the leading effects from $\Delta \alpha\left(M_{\mathrm{Z}}\right)$ and $\Delta \rho$ in the coupling of $\mathrm{W}$ bosons to fermions.

The lowest-order NC cross section is proportional to $\alpha^{2}\left(g_{q q Z}^{\tau}\right)^{2} /\left(c_{\mathrm{w}} s_{\mathrm{w}}\right)^{2}$, where the coupling of the $\mathrm{Z}$ boson to a quark $q$ with chirality $\tau= \pm$ is given by

$$
g_{q q Z}^{\tau}=-\frac{s_{\mathrm{w}}}{c_{\mathrm{w}}} Q_{q}+\frac{I_{q}^{3}}{c_{\mathrm{w}} s_{\mathrm{w}}} \delta_{\tau-},
$$

with $I_{q}^{3}= \pm \frac{1}{2}$ denoting the third component of the weak isospin of a left-handed quark. In order to absorb corrections from $\Delta \rho$, we define the modified coupling factors

$$
\bar{g}_{q q Z}^{\tau}=\left.g_{q q Z}^{\tau}\right|_{s_{\mathrm{w}} \rightarrow \bar{s}_{\mathrm{w}}, c_{\mathrm{w}} \rightarrow \bar{c}_{\mathrm{w}}} .
$$

The $\mathcal{O}(\alpha)$-corrected $\mathrm{NC}$ cross sections, including the leading two-loop improvements, thus, read

$$
\begin{aligned}
& \left.\sigma_{\mathrm{NC}, 1+\mathrm{LL}^{2}}^{\tau}\right|^{\alpha(0)}=\left.\sigma_{\mathrm{NC}, 0}^{\tau}\right|^{\alpha(0)}\left[\left(\frac{\bar{g}_{q q Z}^{\tau} c_{\mathrm{w}} s_{\mathrm{w}}}{g_{q q Z}^{\tau} \bar{c}_{\mathrm{w}} \bar{s}_{\mathrm{w}}}\right)^{2} \frac{1}{(1-\Delta \alpha)^{2}}+\left.\delta_{\mathrm{NC}, 1}^{\tau}\right|^{\alpha(0)}-2 \Delta \alpha\right. \\
& \left.-\left.2 \Delta \rho_{1}\right|^{\alpha(0)}\left(1-\frac{c_{\mathrm{w}} I_{q}^{3}}{g_{q q Z}^{\tau} s_{\mathrm{w}}^{3}} \delta_{\tau-}\right)\right] \\
& =\left.\sigma_{\mathrm{NC}, 0}^{\tau}\right|^{\alpha(0)}\left[1+\left.\delta_{\mathrm{NC}, 1}\right|^{\alpha(0)}-2\left(\left.\Delta \rho_{1}\right|^{\alpha(0)}-\Delta \rho\right)\left(1-\frac{c_{\mathrm{w}} I_{q}^{3}}{g_{q q Z}^{\tau} s_{\mathrm{w}}^{3}} \delta_{\tau-}\right)\right. \\
& +3 \Delta \alpha^{2}+4 \Delta \alpha \Delta \rho\left(1-\frac{c_{\mathrm{w}} I_{q}^{3}}{g_{q q Z}^{\tau} s_{\mathrm{w}}^{3}} \delta_{\tau-}\right) \\
& \left.+\Delta \rho^{2}\left(3+\frac{c_{\mathrm{w}}^{2}}{4\left(g_{q q Z}^{\tau}\right)^{2} s_{\mathrm{w}}^{6}} \delta_{\tau-}+\frac{2 c_{\mathrm{w}}\left(1-3 s_{\mathrm{w}}^{2}\right) I_{q}^{3}}{g_{q q Z}^{\tau} s_{\mathrm{w}}^{5}} \delta_{\tau-}\right)\right] \\
& +\ldots, \\
& \left.\sigma_{\mathrm{NC}, 1+\mathrm{LL}^{2}}^{\tau}\right|^{\alpha\left(M_{\mathrm{Z}}\right)}=\left.\sigma_{\mathrm{NC}, 0}^{\tau}\right|^{\alpha\left(M_{\mathrm{Z}}\right)}\left[\left(\frac{\bar{g}_{q q Z}^{\tau} c_{\mathrm{w}} s_{\mathrm{W}}}{g_{q q Z}^{\tau} \bar{c}_{\mathrm{w}} \bar{s}_{\mathrm{W}}}\right)^{2}+\left.\delta_{\mathrm{NC}, 1}^{\tau}\right|^{\alpha\left(M_{\mathrm{Z}}\right)}\right.
\end{aligned}
$$




$$
\begin{aligned}
& \left.-\left.2 \Delta \rho_{1}\right|^{\alpha\left(M_{\mathrm{Z}}\right)}\left(1-\frac{c_{\mathrm{w}} I_{q}^{3}}{g_{q q Z}^{\tau} s_{\mathrm{w}}^{3}} \delta_{\tau-}\right)\right] \\
& =\left.\sigma_{\mathrm{NC}, 0}^{\tau}\right|^{\alpha\left(M_{\mathrm{Z}}\right)}\left[1+\left.\delta_{\mathrm{NC}, 1}\right|^{\alpha\left(M_{\mathrm{Z}}\right)}-2\left(\left.\Delta \rho_{1}\right|^{\alpha\left(M_{\mathrm{Z}}\right)}-\Delta \rho\right)\left(1-\frac{c_{\mathrm{w}} I_{q}^{3}}{g_{q q Z}^{\tau} s_{\mathrm{w}}^{3}} \delta_{\tau-}\right)\right. \\
& \left.+\Delta \rho^{2}\left(3+\frac{c_{\mathrm{w}}^{2}}{4\left(g_{q q Z}^{\tau}\right)^{2} s_{\mathrm{w}}^{6}} \delta_{\tau-}+\frac{2 c_{\mathrm{w}}\left(1-3 s_{\mathrm{w}}^{2}\right) I_{q}^{3}}{g_{q q Z}^{\tau} s_{\mathrm{w}}^{5}} \delta_{\tau-}\right)\right] \\
& +\ldots, \\
& \left.\sigma_{\mathrm{NC}, 1+\mathrm{LL}^{2}}^{\tau}\right|^{G_{\mu}}=\left.\sigma_{\mathrm{NC}, 0}^{\tau}\right|^{G_{\mu}}\left[\left(\frac{\bar{g}_{q q Z}^{\tau} c_{\mathrm{w}} \bar{s}_{\mathrm{w}}}{g_{q q Z}^{\tau} \bar{c}_{\mathrm{w}} s_{\mathrm{w}}}\right)^{2}+\left.\delta_{\mathrm{NC}, 1}^{\tau}\right|^{G_{\mu}}-\left.\frac{2}{s_{\mathrm{w}}^{2}} \Delta \rho_{1}\right|^{G_{\mu}}\left(1-\frac{c_{\mathrm{w}} I_{q}^{3}}{g_{q q Z}^{\tau} s_{\mathrm{w}}} \delta_{\tau-}\right)\right] \\
& =\left.\sigma_{\mathrm{NC}, 0}^{\tau}\right|^{G_{\mu}}\left[1+\left.\delta_{\mathrm{NC}, 1}^{\tau}\right|^{G_{\mu}}-\frac{2}{s_{\mathrm{w}}^{2}}\left(\left.\Delta \rho_{1}\right|^{G_{\mu}}-\Delta \rho\right)\left(1-\frac{c_{\mathrm{w}} I_{q}^{3}}{g_{q q Z}^{\tau} s_{\mathrm{w}}} \delta_{\tau-}\right)\right] \\
& +\frac{\Delta \rho^{2}}{s_{\mathrm{w}}^{4}}\left(1+2 s_{\mathrm{w}}^{2}+\frac{c_{\mathrm{w}}^{2}}{4\left(g_{q q Z}^{\tau}\right)^{2} s_{\mathrm{w}}^{2}} \delta_{\tau-}-\frac{2 c_{\mathrm{w}}\left(1+s_{\mathrm{w}}^{2}\right) I_{q}^{3}}{g_{q q Z}^{\tau} s_{\mathrm{w}}} \delta_{\tau-}\right) \\
& +\ldots
\end{aligned}
$$

\section{Higher-order final-state radiation off muons}

In the $\mathrm{CC}$ reaction, where a muon appears in the final state, the emission of photons collinear to the outgoing muon leads to corrections that are enhanced by large logarithms of the form $\alpha \ln \left(m_{\mu}^{2} / Q^{2}\right)$ with $Q$ denoting a scale characteristic for the process. The KLN theorem [25] guarantees that these logarithms cancel if photons collinear to the muon are treated fully inclusively. Since we, however, apply a phase-space cut on the energy of the outgoing muon (or equivalently on the remaining total hadronic+photonic energy), contributions enhanced by these logarithms survive. The first-order logarithm $\alpha \ln \left(m_{\mu}^{2} / Q^{2}\right)$ is, of course, implicitly contained in the full $\mathcal{O}(\alpha)$ correction, so that $Q$ is unambiguously fixed in this order. However, it is desirable to control the logarithmically enhanced corrections beyond $\mathcal{O}(\alpha)$. This can be done in the so-called structure function approach [26], where these logarithms are derived from the universal factorization of the related mass singularity. The incorporation of the mass-singular logarithms takes the form of a convolution integral over the lowest-order cross section $\sigma_{\mathrm{CC}, 0}$,

$$
\sigma_{\mathrm{CC}, \mathrm{LLFSR}}=\int \mathrm{d} \sigma_{\mathrm{CC}, 0}\left(p_{l}, p_{q} ; k_{l}, k_{q}\right) \int_{0}^{1} \mathrm{~d} z \Gamma_{\mu \mu}^{\mathrm{LL}}\left(z, Q^{2}\right) \Theta_{\text {cut }}\left(z k_{l}\right),
$$

where the step function $\Theta_{\text {cut }}$ is equal to 1 if the event passes the cut on the rescaled muon momentum $z k_{l}$ and 0 otherwise. The variable $z$ is the momentum fraction describing the muon energy loss by collinear photon emission. This treatment, thus, corresponds to the experimental situation in a fixed-target experiment where photons in the final state are not distinguished from the hadrons that shower in the detector, but the muon is analyzed as an isolated particle. For the structure function $\Gamma_{\mu \mu}^{\mathrm{LL}}\left(z, Q^{2}\right)$ we take into account terms up to $\mathcal{O}\left(\alpha^{3}\right)$ improved by the well-known exponentiation of the soft-photonic parts [26],

$$
\Gamma_{\mu \mu}^{\mathrm{LL}}\left(z, Q^{2}\right)=\frac{\exp \left(-\frac{1}{2} \beta_{\mu} \gamma_{\mathrm{E}}+\frac{3}{8} \beta_{\mu}\right)}{\Gamma\left(1+\frac{1}{2} \beta_{\mu}\right)} \frac{\beta_{\mu}}{2}(1-z)^{\frac{\beta_{\mu}}{2}-1}-\frac{\beta_{\mu}}{4}(1+z)
$$




$$
\begin{aligned}
& -\frac{\beta_{\mu}^{2}}{32}\left\{\frac{1+3 z^{2}}{1-z} \ln (z)+4(1+z) \ln (1-z)+5+z\right\} \\
& -\frac{\beta_{\mu}^{3}}{384}\left\{(1+z)\left[6 \operatorname{Li}_{2}(z)+12 \ln ^{2}(1-z)-3 \pi^{2}\right]\right. \\
& +\frac{1}{1-z}\left[\frac{3}{2}\left(1+8 z+3 z^{2}\right) \ln (z)+6(z+5)(1-z) \ln (1-z)\right. \\
& \quad+12\left(1+z^{2}\right) \ln (z) \ln (1-z)-\frac{1}{2}\left(1+7 z^{2}\right) \ln ^{2}(z) \\
& \left.\left.\quad+\frac{1}{4}\left(39-24 z-15 z^{2}\right)\right]\right\} \\
& +\frac{\beta_{\mu} \beta_{\mathrm{e}}}{48}\left(\frac{1+z^{2}}{1-z}\right)_{+}
\end{aligned}
$$

with $\gamma_{E}$ and $\Gamma(y)$ denoting Euler's constant and the Gamma function, respectively, and the logarithms

$$
\beta_{\mu}=\frac{2 \alpha(0)}{\pi}\left[\ln \left(\frac{Q^{2}}{m_{\mu}^{2}}\right)-1\right], \quad \beta_{\mathrm{e}}=\frac{2 \alpha(0)}{\pi} \ln \left(\frac{Q^{2}}{m_{\mathrm{e}}^{2}}\right) .
$$

The parts solely proportional to a power of $\beta_{\mu}$ correspond to collinear (multi-)photon emission off the muon, the exponential factor describing resummed soft-photonic effects. The non-logarithmic term " -1 " in $\beta_{\mu}$ accounts for a non-singular universal soft-photonic correction. The part proportional to $\beta_{\mu} \beta_{\mathrm{e}}$ corresponds to collinear one-photon emission followed by a collinear splitting $\gamma \rightarrow \mathrm{e}^{+} \mathrm{e}^{-}$. Treating this contribution as part of $\Gamma_{\mu \mu}^{\mathrm{LL}}\left(z, Q^{2}\right)$ means that the event selection is based on a muon momentum $z k_{l}$ also in this case, i.e. that the $\mathrm{e}^{+} \mathrm{e}^{-}$are considered as part of the hadronic and photonic shower in the detector. In this context one should also worry about the possibility of $\mu^{+} \mu^{-}$pairs in the final state resulting from photon splitting. If one assumes that such muons escape the detector, i.e. are not part of the hadronic shower, the corresponding logarithmic contributions cancel, since the full $z$ range is integrated over.

Technically we add the cross section (4.1) to the one-loop result and subtract the lowest-order and one-loop contributions $\mathrm{d} \sigma_{\mathrm{CC}, \mathrm{LL}{ }^{1} \mathrm{FSR}}$ already contained within this formula,

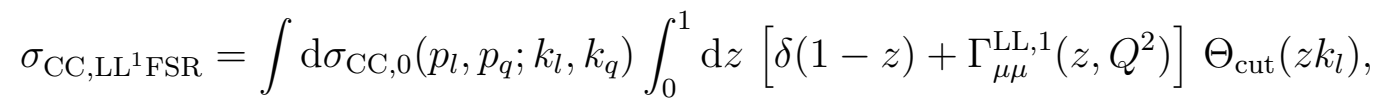

in order to avoid double counting. The one-loop contribution to the structure function reads

$$
\Gamma_{\mu \mu}^{\mathrm{LL}, 1}\left(z, Q^{2}\right)=\frac{\beta_{\mu}}{4}\left(\frac{1+z^{2}}{1-z}\right)_{+} .
$$

More precisely, we adapt the value of $\alpha$ in $\Gamma_{\mu \mu}^{\mathrm{LL}, 1}\left(z, Q^{2}\right)$ to the chosen IPS, so that the $\alpha \ln \left(m_{\mu}^{2} / Q^{2}\right)$ contribution of the $\mathcal{O}(\alpha)$ correction is subtracted exactly. Thus, the procedure of adding higher-order FSR effectively changes also the value of $\alpha$ in the $\alpha \ln \left(m_{\mu}^{2} / Q^{2}\right)$ term to $\alpha(0)$ which is the appropriate coupling for real-photonic effects. 
Finally, note that the uncertainty that is connected with the choice of $Q^{2}$ enters in $\mathcal{O}\left(\alpha^{2}\right)$, since all $\mathcal{O}(\alpha)$ corrections, including constant terms, are taken into account. As default we choose the value

$$
Q=\max \left\{\xi \sqrt{s}, 2 m_{\mu}\right\}
$$

with $\xi=1$. In order to quantify the scale uncertainty, we vary $\xi$ between 0.3 and 3 . The lower limit $2 m_{\mu}$ accounts for the fact that the muon energy $\sqrt{s} / 2$ in the CM frame cannot drop below $m_{\mu}$.

\section{$5 \quad$ Parton distribution functions}

\subsection{Inclusion of $\mathcal{O}(\alpha)$ corrections}

The inclusion of $\mathcal{O}(\alpha)$ corrections to hadronic cross sections, which is described in the following in detail, conceptually proceeds along the same lines as the incorporation of next-to-leading (NLO) QCD corrections.

The $\mathcal{O}(\alpha)$-corrected parton cross sections contain mass singularities of the form $\alpha \ln \left(m_{q}\right)$, which are due to collinear photon radiation off the initial-state quarks or due to a collinear splitting $\gamma \rightarrow q \bar{q}$ for initial-state photons. In complete analogy to factorization in NLO QCD calculations, we absorb these collinear singularities into the quark and photon distributions. For processes that involve only quarks and/or antiquarks in the initial state in lowest order, the factorization is achieved by replacing the (anti-)quark distribution $q(x)$ according to

$$
\begin{aligned}
q(x) \rightarrow q(x, & \left.M^{2}\right)-\int_{x}^{1} \frac{\mathrm{d} z}{z} q\left(\frac{x}{z}, M^{2}\right) \frac{\alpha}{2 \pi} Q_{q}^{2} \\
& \times\left\{\ln \left(\frac{M^{2}}{m_{q}^{2}}\right)\left[P_{f f}(z)\right]_{+}-\left[P_{f f}(z)(2 \ln (1-z)+1)\right]_{+}+C_{f f}(z)\right\} \\
& -\int_{x}^{1} \frac{\mathrm{d} z}{z} \gamma\left(\frac{x}{z}, M^{2}\right) \frac{\alpha}{2 \pi} 3 Q_{q}^{2}\left\{\ln \left(\frac{M^{2}}{m_{q}^{2}}\right) P_{f \gamma}(z)+C_{f \gamma}(z)\right\}
\end{aligned}
$$

where $M$ is the factorization scale, $C_{i j}(z)$ are the so-called coefficient functions, and the splitting functions $P_{i j}(z)$ are defined as

$$
P_{f f}(z)=\frac{1+z^{2}}{1-z}, \quad P_{f \gamma}(z)=z^{2}+(1-z)^{2} .
$$

The replacement (5.1) defines the same finite parts in the $\mathcal{O}(\alpha)$ correction, i.e. the same coefficient functions, as the usual $D$-dimensional regularization for exactly massless partons, where the $\ln \left(m_{q}\right)$ terms appear as $1 /(D-4)$ poles. We have derived this correspondence by explicit calculation and found agreement with the results of Ref. [27]. The actual form of the coefficient functions defines the finite parts of the $\mathcal{O}(\alpha)$ corrections and, thus, the factorization scheme. Following standard definitions of QCD, we distinguish the $\overline{\mathrm{MS}}$ and DIS-like schemes which are formally defined by

$$
\begin{aligned}
C_{f f}^{\overline{\mathrm{MS}}}(z) & =C_{f \gamma}^{\overline{\mathrm{MS}}}(z)=0, \\
C_{f f}^{\mathrm{DIS}}(z) & =\left[P_{f f}(z)\left(\ln \left(\frac{1-z}{z}\right)-\frac{3}{4}\right)+\frac{9+5 z}{4}\right]_{+}, \\
C_{f \gamma}^{\mathrm{DIS}}(z) & =P_{f \gamma}(z) \ln \left(\frac{1-z}{z}\right)-8 z^{2}+8 z-1 .
\end{aligned}
$$


The $\overline{\mathrm{MS}}$ scheme is motivated by formal simplicity, because it merely rearranges the UVdivergent terms (plus some trivial constants) as defined in dimensional regularization. The DIS-like scheme is defined in such a way that the DIS structure function $F_{2}$ does not receive any corrections; in other words, the radiative corrections to electron-proton DIS are implicitly contained in the PDFs in this case. Whatever scheme has been adopted in the extraction of PDFs from experimental data, the same scheme has to be used when predictions for other experiments are made using these PDFs.

The absorption of the collinear singularities of $\mathcal{O}(\alpha)$ into PDFs requires the inclusion of the corresponding $\mathcal{O}(\alpha)$ corrections into the Dokshitzer-Gribov-Lipatov-Altarelli-Parisi (DGLAP) evolution of these distributions and into their fit to experimental data. In the past, when the $\mathcal{O}(\alpha)$ effects were not yet included in the PDF fit to data, the inclusion of the $\mathcal{O}(\alpha)$ corrections to the DGLAP evolution showed [28] that the impact of these corrections on the quark distributions in the $\overline{\mathrm{MS}}$ factorization scheme should be smaller than remaining QCD uncertainties. Therefore, $\mathcal{O}(\alpha)$ corrections to hadronic processes were usually included in the $\overline{\mathrm{MS}}$ factorization scheme using the existing PDFs, which ignored $\mathcal{O}(\alpha)$ effects. This was also done in our previous calculation [15].

Recently the MRST collaboration released the first set of PDFs [10], called "MRST2004QED", that consistently include $\mathcal{O}(\alpha)$ corrections. The $\mathcal{O}(\alpha)$ effects induce two important modifications in the PDFs. Firstly, the difference in the electric charges of the up- and down-type quarks leads to a violation of the isospin symmetry between proton and neutron PDFs. It was frequently pointed out in the literature $[4,5,7-10]$ that this isospin violation, i.e. $u^{\mathrm{P}}(x) \neq d^{\mathrm{n}}(x)$ etc., is of particular importance when analyzing ratios of neutral-current to charged-current cross sections in neutrino DIS. Secondly, the factorization of collinear singularities from photon emission leads to photon distribution functions both for the proton and the neutron. The corresponding photon-induced contributions to hadronic cross sections have not yet been considered in the literature (since the photon PDF was not yet available), but have been assumed to be small, because of an implicit suppression factor $\alpha$ in the radiatively induced photon PDF. In our discussion of numerical results below we illustrate the size of the $\mathcal{O}(\alpha)$ corrections to the PDFs in neutrino DIS cross sections by switching these corrections on and off. Moreover, we calculate and discuss the size of the photon-induced contributions to the cross sections explicitly. Some technical details of this part of the calculation are given in the Appendix.

The authors of the MRST2004QED set of PDFs did not explicitly state in Ref. [10] which scheme is relevant for the factorization of the $\mathcal{O}(\alpha)$ corrections. They themselves did not include any $\mathcal{O}(\alpha)$ corrections, neither in the underlying data set nor in the used theoretical predictions, and used the same data as in their standard fit, implicitly assuming that no $\mathcal{O}(\alpha)$ corrections corresponding to photon emission off incoming quarks had been applied in the data analysis. For the $F_{2}$ data analysis at HERA this assumption was confirmed to us by members of the $\mathrm{H} 1$ and ZEUS collaborations and by theorists involved in the analysis [29]. Therefore, we conclude that the $\mathcal{O}(\alpha)$ corrections induced by photon emission off incoming quarks implicitly went into the PDFs in the fit, which thus precisely corresponds to the DIS-like factorization. We use this scheme in the numerical analysis below. We note, however, that the MRST2004QED PDFs are defined in NLO QCD in the $\overline{\mathrm{MS}}$ factorization scheme. 


\subsection{Nuclear structure functions}

In reasonable approximation, nuclear structure functions can be constructed from the PDFs for protons and neutrons upon appropriately weighting the distribution for a specific parton according to the probability of finding a proton or neutron in the target. The NuTeV collaboration, e.g., quotes a neutron excess of $\epsilon=(N-Z) /(N+Z)=5.74 \%$ in their target, where $N$ and $Z$ are the (average) numbers of neutrons and protons in the target, respectively. The probabilities $c_{\mathrm{p}}$ and $c_{\mathrm{n}}$ to find a proton or neutron, are thus given by

$$
c_{\mathrm{p}}=\frac{1}{2}(1-\epsilon), \quad c_{\mathrm{n}}=\frac{1}{2}(1+\epsilon) .
$$

The corresponding nuclear structure functions read

$$
f_{a}(x)=c_{\mathrm{p}} a^{\mathrm{p}}(x)+c_{\mathrm{n}} a^{\mathrm{n}}(x), \quad a=q, \bar{q}, \gamma,
$$

where $a^{\mathrm{p}}(x)$ and $a^{\mathrm{n}}(x)$ generically denote the PDFs for the proton and neutron, respectively. An isoscalar target obviously corresponds to the choice $\epsilon=0$, i.e. $c_{\mathrm{p}}=c_{\mathrm{n}}=\frac{1}{2}$. In the following we make use of nuclear structure functions derived with the value for the neutron excess $\epsilon$ quoted by $\mathrm{NuTeV}$.

\section{Discussion of results}

\subsection{Input parameters and setup of the calculation}

For the numerical evaluation we use the following set of SM parameters,

$$
\begin{array}{rlrlrl}
G_{\mu} & =1.16637 \times 10^{-5} \mathrm{GeV}^{-2}, & \alpha(0) & =1 / 137.03599911, & \alpha\left(M_{\mathrm{Z}}\right) & =1 / 128.93, \\
M_{\mathrm{W}} & =80.425 \mathrm{GeV}, & M_{\mathrm{Z}} & =91.1876 \mathrm{GeV}, & \alpha_{\mathrm{s}} & =0.1172, \\
M_{\mathrm{H}} & =115 \mathrm{GeV}, & & & \\
m_{\mathrm{e}}=0.51099892 \mathrm{MeV}, & m_{\mu}=105.658369 \mathrm{MeV}, & m_{\tau}=1.77699 \mathrm{GeV}, \\
m_{\mathrm{u}}=66 \mathrm{MeV}, & m_{\mathrm{c}}=1.2 \mathrm{GeV}, & m_{\mathrm{t}}=178 \mathrm{GeV}, \\
m_{\mathrm{d}}=66 \mathrm{MeV}, & m_{\mathrm{s}}=150 \mathrm{MeV}, & m_{\mathrm{b}}=4.3 \mathrm{GeV},
\end{array}
$$

which essentially follows Ref. [30]. For the top-quark mass $m_{\mathrm{t}}$ we have taken the value of Ref. [31]. The masses of the light quarks are adjusted to reproduce the hadronic contribution to the photonic vacuum polarization of Ref. [32].

As explained in detail in Section 5.1, we consistently use the set of MRST2004QED PDFs introduced in Ref. [10], which include $\mathcal{O}(\alpha)$ corrections. In order to quantify the modifications induced by these corrections to the PDFs we use the alternative set MRST2004QEDx, which we got from the MRST collaboration and which differs from the original MRST2004QED set only by neglecting the $\mathcal{O}(\alpha)$ effects. Since we do not include NLO QCD corrections in our calculation, it does not make sense to discuss the factorization scale dependence of our results, because the QED and QCD factorization scales are set equal in the MRST2004QED PDFs. As already done in Ref. [15], we set the QED factorization scale $M^{2}$ to the momentum transfer of the leptonic line; the only 
difference comes from the fact that the MRST2004QED PDFs are not delivered for $M$ values below their start scale $M_{0}$. Therefore, we use

$$
M^{2}=\max \left\{-\left(p_{l}-k_{l}\right)^{2}, M_{0}^{2}\right\}, \quad M_{0}^{2}=1.25 \mathrm{GeV}^{2},
$$

where $p_{l}$ and $k_{l}$ are the incoming neutrino momentum and the outgoing neutrino or lepton momentum, respectively. Moreover, the PDFs require $x>10^{-5}$; otherwise they are set to zero.

The energy of the incoming neutrinos is set to $E_{\nu}^{\mathrm{LAB}}=80 \mathrm{GeV}$, but the energy of all outgoing hadrons and photons is constrained by $E_{\mathrm{had}+\text { phot }}^{\mathrm{LAB}}>20 \mathrm{GeV}$, which differs from the setup used in Ref. [15].

As done in Refs. [14,15], we quantify the corrections to the $R^{\nu}$ ratio by

$$
\delta R_{\mathrm{NC}}^{\nu}=\frac{\delta \sigma_{\mathrm{NC}}^{\nu}}{\sigma_{\mathrm{NC}}^{\nu}}, \quad \delta R_{\mathrm{CC}}^{\nu}=-\frac{\delta \sigma_{\mathrm{CC}}^{\nu}}{\sigma_{\mathrm{CC}}^{\nu}}
$$

where $\delta \sigma_{\mathrm{NC} / \mathrm{CC}}^{\nu}$ are the corrections to the $\mathrm{NC}$ and $\mathrm{CC}$ cross sections. These corrections, to a reasonable approximation, translate into a shift in the extracted on-shell weak mixing angle $\sin ^{2} \theta_{\mathrm{W}}$ according to $[14,15]$

$$
\Delta \sin ^{2} \theta_{\mathrm{W}}=\frac{\frac{1}{2}-\sin ^{2} \theta_{\mathrm{W}}+\frac{20}{27} \sin ^{4} \theta_{\mathrm{W}}}{1-\frac{40}{27} \sin ^{2} \theta_{\mathrm{W}}}\left(\delta R_{\mathrm{NC}}^{\nu}+\delta R_{\mathrm{CC}}^{\nu}\right) .
$$

Technically we have extended our $\mathcal{O}(\alpha)$ calculation w.r.t. its description in Ref. [15] by implementing a generalization of the dipole subtraction formalism to non-collinear-safe observables. ${ }^{1}$ All numerical results below are obtained with this technique, which turns out to be more efficient, but they have been confirmed by phase-space slicing as described in Ref. [15].

\subsection{Numerical results on integrated cross sections}

Table 1 summarizes our numerical results as obtained in the three different IPS " $\alpha(0)$ ", " $\alpha\left(M_{\mathrm{Z}}\right)$ ", and " $G_{\mu}$ ". In the first row, denoted " $\mathcal{O}(\alpha)$, PDFqed", we give the cross-section ratio $R_{0}^{\nu}$ in lowest order as well as the corrections $\delta R_{\mathrm{NC}}^{\nu}, \delta R_{\mathrm{CC}}^{\nu}, \Delta \sin ^{2} \theta_{\mathrm{W}}$ based on $\mathcal{O}(\alpha)$ corrections only. The results are based on the $\mathcal{O}(\alpha)$-corrected PDFs MRST2004QED [10], but contributions from the $\gamma$-induced processes are not taken into account in this row. This means, apart from the different kinematical setup, the new PDFs (with DIS-like QED factorization), and the new input parameters, the first row is based on the calculation of $\mathcal{O}(\alpha)$ corrections described in Ref. [15]. The second row, denoted "OOO $(\alpha)$, PDFqedx", contains the corresponding results obtained with the set of PDFs MRST2004QEDx that differ from MRST2004QED only in the consistent neglect of the $\mathcal{O}(\alpha)$ corrections in the PDFs. Comparing the first two rows for each IPS, the influence of the implicit $\mathcal{O}(\alpha)$ corrections in the PDFs can be quantified to about 0.0004 in $\sin ^{2} \theta_{\mathrm{W}}$. All the remaining rows in Table 1 are calculated with the $\mathcal{O}(\alpha)$-corrected PDFs MRST2004QED.

\footnotetext{
${ }^{1}$ The dipole subtraction formalism as described for real QCD corrections [40] and photon radiation $[34,35]$ is originally set up for collinear-safe observables where all final-state mass singularities cancel. The generalization to non-collinear-safe observables is partially described (for charged final-state particles only) in Ref. [36]; the complete generalization will be published elsewhere [37].
} 


\begin{tabular}{|c|c|c|c|c|c|}
\hline IPS & contribution & $R_{0}^{\nu}$ & $\delta R_{\mathrm{NC}}^{\nu}$ & $\delta R_{\mathrm{CC}}^{\nu}$ & $\Delta \sin ^{2} \theta_{\mathrm{W}}$ \\
\hline \multirow[t]{8}{*}{$\alpha(0)$} & $\mathcal{O}(\alpha)$, PDFqed & 0.30455 & 0.0562 & -0.0840 & -0.0130 \\
\hline & $\mathcal{O}(\alpha)$, PDFqedx & 0.31167 & 0.0555 & -0.0841 & -0.0134 \\
\hline & univ. 2-loop & & 0.01275 & -0.01279 & -0.00002 \\
\hline & univ. 2-loop' & & 0.01229 & -0.01235 & -0.00003 \\
\hline & h.o. FSR & & 0 & $0.000014(1)$ & $0.000007(1)$ \\
\hline & h.o. FSR' & & 0 & 0.000010 & 0.000005 \\
\hline & $\gamma$ process & & -0.000005 & -0.000063 & -0.000032 \\
\hline & "best" & & 0.0690 & -0.0968 & -0.0130 \\
\hline \multirow[t]{8}{*}{$\alpha\left(M_{\mathrm{Z}}\right)$} & $\mathcal{O}(\alpha)$, PDFqed & 0.30455 & -0.0660 & 0.0365 & -0.0138 \\
\hline & $\mathcal{O}(\alpha)$, PDFqedx & 0.31167 & -0.0668 & 0.0365 & -0.0142 \\
\hline & univ. 2-loop & & 0.01248 & -0.01319 & -0.00033 \\
\hline & univ. 2-loop' & & 0.01258 & -0.01330 & -0.00033 \\
\hline & h.o. FSR & & 0 & $0.00073(1)$ & $0.00034(1)$ \\
\hline & h.o. FSR' & & 0 & 0.00072 & 0.00034 \\
\hline & $\gamma$ process & & -0.000005 & -0.000063 & -0.000032 \\
\hline & "best" & & -0.0535 & 0.0240 & -0.0138 \\
\hline \multirow[t]{8}{*}{$G_{\mu}$} & $\mathcal{O}(\alpha)$, PDFqed & 0.30455 & 0.0016 & -0.0303 & -0.0135 \\
\hline & $\mathcal{O}(\alpha)$, PDFqedx & 0.31167 & 0.0009 & -0.0303 & -0.0138 \\
\hline & univ. 2-loop & & -0.00039 & 0 & -0.00018 \\
\hline & univ. 2-loop' & & -0.00039 & 0 & -0.00018 \\
\hline & h.o. FSR & & 0 & $0.00040(1)$ & $0.00019(1)$ \\
\hline & h.o. FSR' & & 0 & 0.00039 & 0.00018 \\
\hline & $\gamma$ process & & -0.000005 & -0.000063 & -0.000032 \\
\hline & "best" & & 0.0012 & -0.0299 & -0.0135 \\
\hline
\end{tabular}

Table 1: Results on the ratio $R^{\nu}$ with $E_{\nu}^{\mathrm{LAB}}=80 \mathrm{GeV}, E_{\text {had }+ \text { phot }}^{\mathrm{LAB}}>20 \mathrm{GeV}$ in lowest order $\left(R_{0}^{\nu}\right)$, corrections from $\mathrm{NC}$ and $\mathrm{CC}$ cross sections $\left(\delta R_{\mathrm{NC}}^{\nu}\right.$ and $\left.\delta R_{\mathrm{CC}}^{\nu}\right)$, and shift $\Delta \sin ^{2} \theta_{\mathrm{W}}$, for various input-parameter schemes (IPS). All but the "PDFqedx" numbers are obtained with the $\mathcal{O}(\alpha)$-corrected PDFs MRST2004QED; in PDFqedx the $\mathcal{O}(\alpha)$ corrections within the PDFs are switched off. The different rows correspond to the pure " $\mathcal{O}(\alpha)$ " corrections, to improvements by "universal 2-loop" corrections, to "higher-order FSR" corrections, and to contributions from the " $\gamma$ process" with a photon in the initial state. The number in parentheses indicates the statistical integration error in the last digit. For more details, see main text. 


\begin{tabular}{clccc}
\hline IPS & contribution & $\xi$ & $\delta R_{\mathrm{CC}}^{\nu}$ & $\Delta \sin ^{2} \theta_{\mathrm{W}}$ \\
\hline$\alpha(0)$ & h.o. FSR & 0.3 & -0.000008 & -0.000004 \\
& & 1 & 0.000010 & 0.000005 \\
& & 3 & 0.000040 & 0.000019 \\
\hline$\alpha\left(M_{\mathrm{Z}}\right)$ & h.o. FSR & 0.3 & 0.00046 & 0.00021 \\
& & 1 & 0.00072 & 0.00034 \\
& & 3 & 0.00099 & 0.00046 \\
\hline$G_{\mu}$ & h.o. FSR & 0.3 & 0.00024 & 0.00011 \\
& & 1 & 0.00039 & 0.00018 \\
& & 3 & 0.00055 & 0.00026 \\
\hline
\end{tabular}

Table 2: Scale dependence of higher-order FSR corrections.

The rows denoted "univ. 2-loop" and "univ. 2-loop'" show the impact of the universal electroweak corrections beyond $\mathcal{O}(\alpha)$ induced by $\Delta \alpha$ and $\Delta \rho$, as worked out in Section 3. In detail, the first of these rows correspond to the resummed versions of the two-loop effects [first equations in (3.9)-(3.11) and (3.14)-(3.16)], while the second corresponds to the genuine two-loop parts [second equations in (3.9) etc.]. The difference between the two versions is $\lesssim 10^{-5}$ in $\sin ^{2} \theta_{\mathrm{W}}$ and, thus, negligible. Note that the size of these universal two-loop corrections to the cross sections is rather large, more than $1 \%$, in the $\alpha(0)$ and $\alpha\left(M_{\mathrm{Z}}\right)$ schemes. In the $G_{\mu}$ scheme the universal effects beyond $\mathcal{O}(\alpha)$ are very small also for the individual cross sections, reflecting the fact that they are widely absorbed in the lowest order. Since the effects enter CC and NC cross sections in a similar way, they widely cancel in the cross-section ratio and influence $\sin ^{2} \theta_{\mathrm{W}}$ only at the level of 0.0003 , depending on the IPS.

The result on higher-order FSR corrections to the CC cross section are indicated by "h.o. FSR" and "h.o. FSR" in Table 1, where the first of these rows result from the structure function as given in (4.2) and the second from an expansion of (4.2) up to $\mathcal{O}\left(\alpha^{3}\right)$. The differences between these numbers, which quantify leading logarithmic FSR effects beyond $\mathcal{O}\left(\alpha^{3}\right)$, are negligible. The corrections in the $\alpha\left(M_{\mathrm{Z}}\right)$ - and $G_{\mu^{-}}$-schemes are significantly larger than in the $\alpha(0)$-scheme, because in those schemes the higher-order FSR corrections also receive a contribution from the $\mathcal{O}(\alpha)$ part, more precisely from the $\mathcal{O}(\alpha)$ part calculated with $\alpha(0)$ minus the $\mathcal{O}(\alpha)$ part calculated with the $\alpha$ from the chosen IPS. In fact almost the entire effect is due to this change from $\alpha$ to $\alpha(0)$ in the $\mathcal{O}(\alpha)$ FSR, which is $1.14 \%$ in $\delta R_{\mathrm{CC}}^{\nu}$ when calculated with $\alpha(0)$. In the $G_{\mu}$ scheme, higher-order FSR affects $\sin ^{2} \theta_{\mathrm{W}}$ at the level of 0.0002 .

As explained in Section 4, our results depend on the QED splitting scale $Q$ for collinear FSR in $\mathcal{O}\left(\alpha^{2}\right)$ and beyond, while in $\mathcal{O}(\alpha)$ the scale $Q$ is fixed by the non-logarithmic corrections. Table 2 illustrates the residual scale dependence by varying the parameter $\xi$ in (4.6) between 0.3 and 3; the default choice is $\xi=1$. The table shows that in the $\alpha(0)$-scheme the scale uncertainty is of the order of 0.00002 when translated into a shift in $\sin ^{2} \theta_{\mathrm{W}}$. While this number corresponds to genuine FSR effects beyond $\mathcal{O}(\alpha)$, the larger scale dependence in the $\alpha\left(M_{\mathrm{Z}}\right)$ - and $G_{\mu}$-schemes originates from the scale change in the 


\begin{tabular}{ll}
\hline source & $\Delta \sin ^{2} \theta_{\mathrm{W}}$ \\
\hline $\mathcal{O}(\alpha)$ effect in PDFs & 0.0004 \\
\hline univ. electroweak effects & 0.00003 \\
h.o. FSR & 0.00002 \\
non-univ. electroweak effects & 0.0003 \\
\hline
\end{tabular}

Table 3: Estimates of theoretical uncertainties from missing electroweak higher-order effects in the $G_{\mu}$-scheme.

term $[\alpha(0) / \pi-\alpha / \pi] \ln \left(m_{\mu}^{2} / Q^{2}\right)$, which changes the coupling $\alpha$ to $\alpha(0)$ in the $\mathcal{O}(\alpha)$ FSR corrections.

The contributions of the $\gamma$-induced processes are shown in Table 1 in the rows " $\gamma$ process". Since we have set the photon coupling to $\alpha(0)$ in all three IPS, the relative impact of these corrections is identical in all the IPS. The effect on $\sin ^{2} \theta_{\mathrm{W}}$ is about 0.00003 and, thus, negligible.

The numbers called "best" in Table 1 show the sum of the $\mathcal{O}(\alpha)$ and higher-order corrections, where the latter are the sum of the contributions labelled "univ. 2-loop", "h.o. FSR", and " $\gamma$ process". The fact that these "best" numbers agree with the purely $\mathcal{O}(\alpha)$-corrected results up to $\lesssim 0.0001$ in $\sin ^{2} \theta_{\mathrm{W}}$ is accidental, since universal effects and FSR, which widely cancel each other, are of different physical origin. This means, in particular, the cancellation will not take place for a different kinematical setup (neutrino energy, hadronic energy cut, etc.).

Finally, in Table 3 we estimate the theoretical uncertainties from missing electroweak higher-order effects from the results discussed above. Table 1 clearly shows that the $\mathcal{O}(\alpha)$ corrections implicit in the PDFs affects $\sin ^{2} \theta_{\mathrm{W}}$ of the order 0.0004 , which is not negligible w.r.t. the error of about 0.0016 quoted by NuTeV. $\mathcal{O}(\alpha)$-corrected PDFs should therefore be used in such an analysis. If this is done, the error estimate of 0.0004 will of course be reduced. In Table 3 we estimate the missing universal electroweak effects in the $G_{\mu^{-}}$ scheme, which is the most reliable IPS, by changing the parameter $\Delta \rho$ by $2 \%$ of its size, which is of the order of effects beyond two loops [38]. The resulting uncertainty is about 0.00003 and thus negligible. Missing higher-order effects from FSR have been quantified to be of the order 0.00002 in Table 2 above. We take this value from the $\alpha(0)$-scheme, because the larger uncertainties from changing $\alpha$ to $\alpha(0)$ in the two other schemes are part of non-universal electroweak effects beyond $\mathcal{O}(\alpha)$. These non-universal electroweak effects are estimated as follows. In the $G_{\mu}$-scheme, the $\mathcal{O}(\alpha)$ corrections to the $R^{\nu}$ ratio are about $2.9 \%$ where about $1.1 \%$ are due to $\operatorname{FSR}(\xi=1)$. The relative coupling of FSR is clearly $\alpha(0)$, but the effective value of $\alpha$ in the non-universal $1.8 \%$ can only be fixed in a twoloop calculation. Therefore, changing the value of $\alpha_{G_{\mu}}$ to $\alpha(0)$ or $\alpha\left(M_{\mathrm{Z}}\right)$, i.e. varying the $1.8 \%$ by about $3 \%$, should give an idea about missing electroweak two-loop effects. This procedure changes $\sin ^{2} \theta_{\mathrm{W}}$ by 0.0003 , which means that missing non-universal electroweak effects should dominate the theoretical uncertainty from electroweak corrections. 


\subsection{Numerical results on differential cross sections}

Figures 1-3 illustrate the differential cross sections $\mathrm{d} \sigma / \mathrm{d} y$ and $\mathrm{d}^{2} \sigma / \mathrm{d} x / \mathrm{d} y$ and the corresponding corrections. The curves labelled "total" correspond to $\mathrm{d} \sigma / \mathrm{d} y$ shown as histograms, the other curves correspond to $\mathrm{d}^{2} \sigma / \mathrm{d} x / \mathrm{d} y$ integrated over the indicated $x$ range. Note that we actually distinguish between the variable $x$, which is the argument of the PDFs, and the Bjorken variable $x_{\mathrm{Bj}}$, which is reconstructed from initial- and finalstate variables. The lowest-order predictions of Figure 1 show that the integrated cross sections, both for the $\mathrm{NC}$ and $\mathrm{CC}$ channel, are dominated by the $x$ range $\left(x=x_{\mathrm{Bj}}\right.$ in lowest order) $x \lesssim 0.4$, while the distributions in $y$ are flat. The corrections to the NC process (Figure 2) vary between $-0.1 \%$ and $+0.5 \%$ for all $x, x_{\mathrm{Bj}}$, and $y$ values. The distributions in $x$ and $x_{\mathrm{Bj}}$ are almost identical, and the corrections in the various $x$ and $x_{\mathrm{Bj}}$ ranges do not deviate by more than $0.2 \%$ from the correction to the corresponding fully integrated quantity $\mathrm{d} \sigma / \mathrm{d} y$. The situation is very different in the $\mathrm{CC}$ case, as shown in Figure 3. The corrections to $\mathrm{d} \sigma / \mathrm{d} y$ vary within $\sim 2-4 \%$. The upper left plot in Figure 3 demonstrates that the corrections vary very little in $x$, but the upper right plot shows a very large variation in $x_{\mathrm{Bj}}$. This is due to the fact that binning w.r.t. the parton $x$ does not destroy the inclusiveness of collinear FSR off the muon, but binning in $x_{\mathrm{Bj}}$ does. In other words, a cut in $x$ does not cut into the cone of collinear FSR, but a cut in $x_{\mathrm{Bj}}$ does. These features also show up in higher-order FSR as depicted in the lower plots of Figure 3.

The results on the distributions are particularly interesting for the issue of implementing electroweak corrections in Monte Carlo generators upon reweigthing certain distributions. The corrections to the differential CC cross sections suggest that a reweighting in distributions w.r.t. $x$ is less delicate than in $x_{\mathrm{Bj}}$.

\section{Conclusions}

A thorough understanding of electroweak corrections to deep-inelastic neutrino scattering is indispensable for exploiting measurements of $\mathrm{NuTeV}$ and NOMAD to extract a value for the on-shell weak mixing angle $\sin ^{2} \theta_{\mathrm{W}}=1-M_{\mathrm{W}}^{2} / M_{\mathrm{Z}}^{2}$. In this paper, we have supplemented our previous calculation of electroweak $\mathcal{O}(\alpha)$ corrections by higherorder effects, in order to reduce the theoretical uncertainty from unknown electroweak corrections.

We take into account universal two-loop effects from $\Delta \alpha$ and $\Delta \rho$ as well as higher-order final-state photon radiation off muons in the structure function approach. The impact on $\sin ^{2} \theta_{\mathrm{W}}$ of these effects are about 0.0003 ; compared to the experimental uncertainty quoted by $\mathrm{NuTeV}$, which is about 0.0016 , these effects are small.

Moreover, we employ the recently released $\mathcal{O}(\alpha)$-improved parton distributions MRST2004QED and identify the relevant QED factorization scheme, which is DIS like. Switching on and off the implicit $\mathcal{O}(\alpha)$ corrections in the parton distributions, changes $\sin ^{2} \theta_{\mathrm{W}}$ at the level of 0.0004 .

As a technical byproduct, we describe two variants of phase-space slicing and the dipole subtraction method for calculating processes with initial-state photons. Such photoninduced processes become relevant, because the $\mathcal{O}(\alpha)$ evolution of the parton distributions 

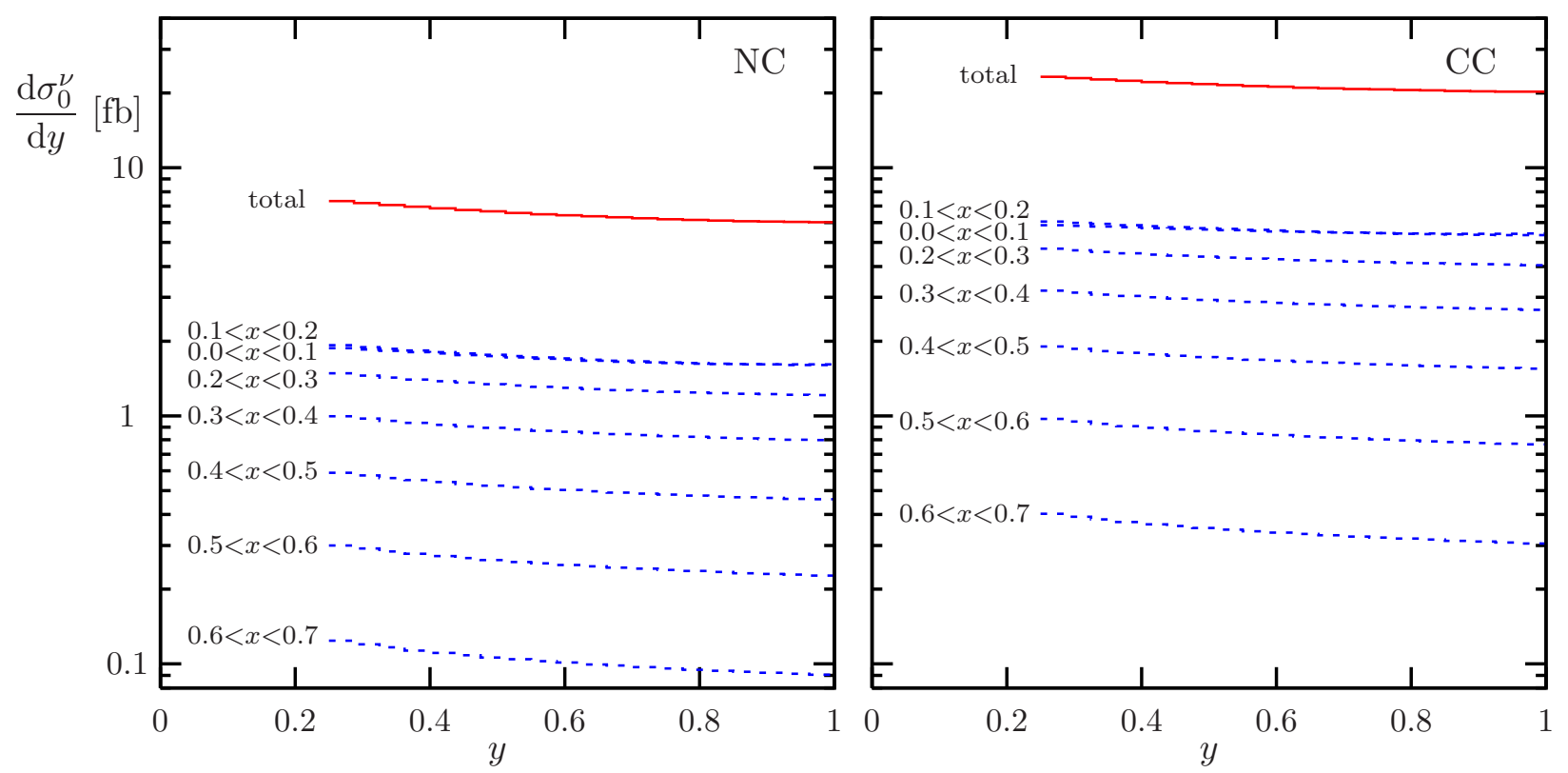

Figure 1: Lowest-order predictions for the distributions in $y$ (total) and individual contributions from different ranges in $x\left(=x_{\mathrm{Bj}}\right.$ in lowest order). The $\mathrm{NC}$ channel for $\nu$ scattering is shown on the l.h.s., the $\mathrm{CC}$ channel on the r.h.s.
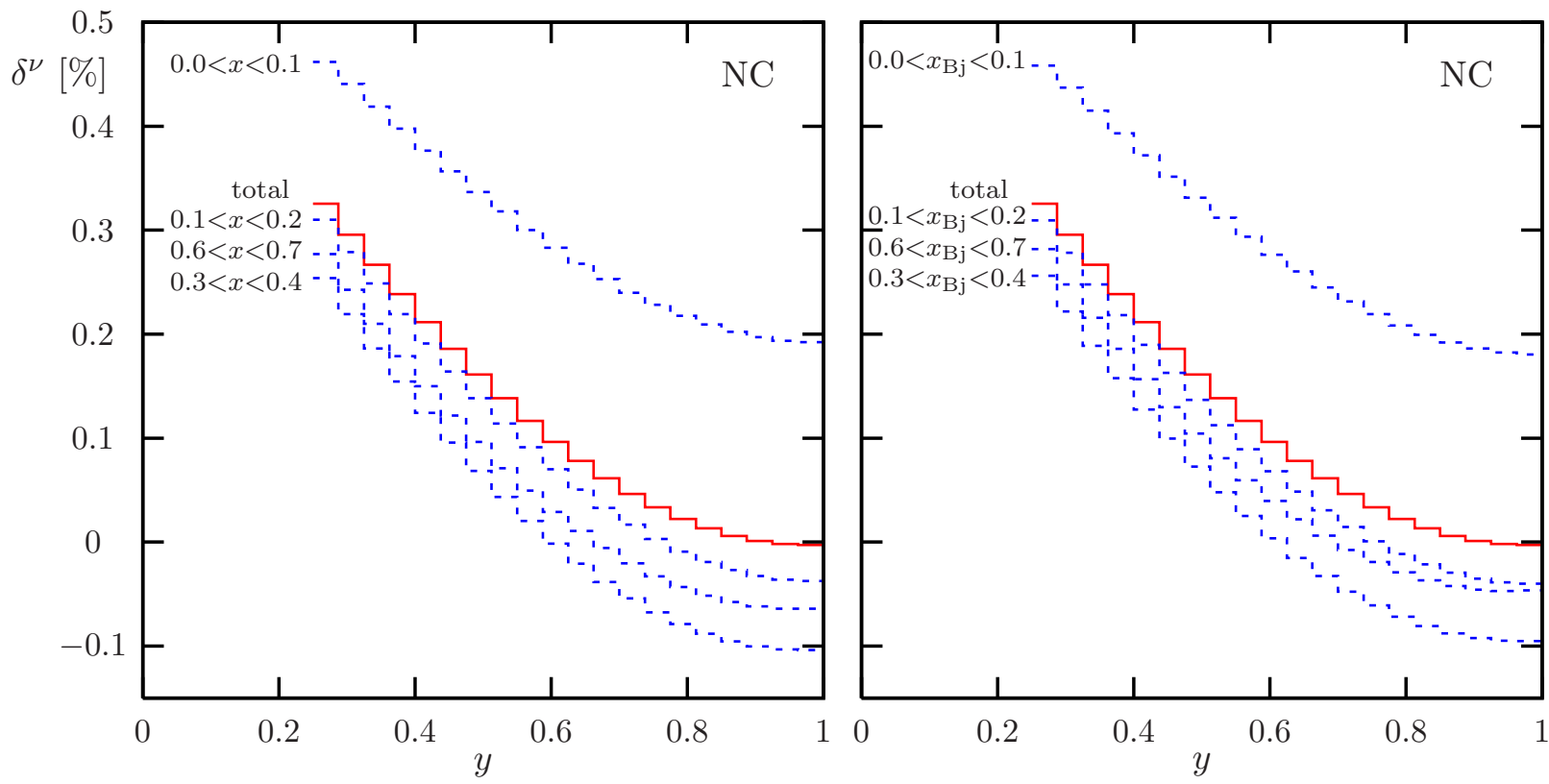

Figure 2: Relative electroweak corrections $\delta^{\nu}=\delta \sigma^{\nu} / \sigma_{0}^{\nu}$ to the distributions in $y$ (total) and to individual contributions from different ranges in $x$ (l.h.s.) and in $x_{\mathrm{Bj}}$ (r.h.s.). 

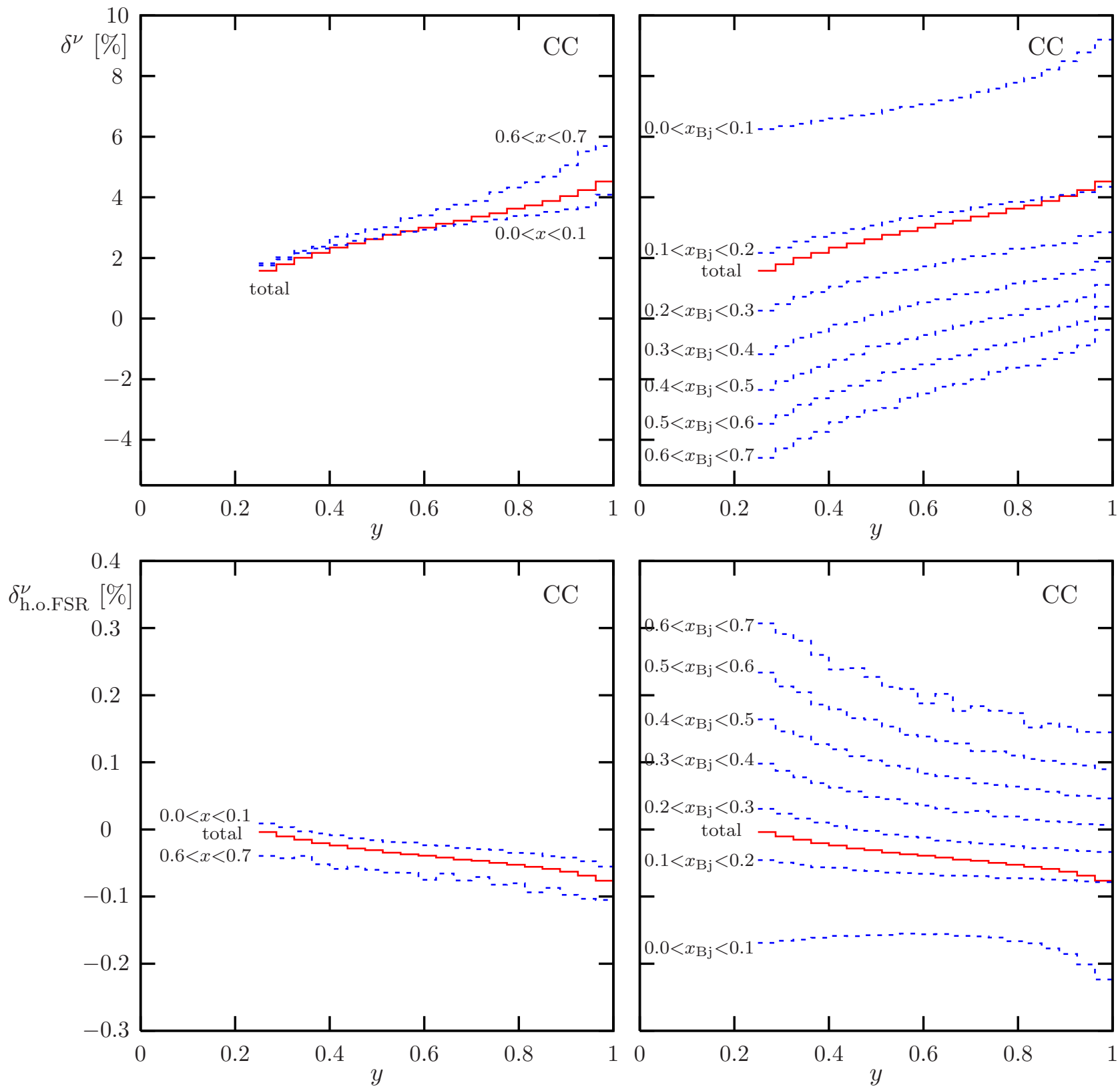

Figure 3: Relative electroweak corrections $\delta^{\nu}=\delta \sigma^{\nu} / \sigma_{0}^{\nu}$ (upper plots) and higher-order FSR (lower plots) to the distributions in $y$ (total) and to individual contributions from different ranges in $x$ (l.h.s.) and in $x_{\mathrm{Bj}}$ (r.h.s.). 
generates a photon distribution. Owing to the smallness of the available scattering energy, the impact of photon-induced processes on $\sin ^{2} \theta_{\mathrm{W}}$ is negligible for $\mathrm{NuTeV}$.

A numerical discussion of the higher-order effects suggests that the remaining theoretical uncertainty from unknown electroweak corrections is dominated by unknown nonuniversal two-loop effects and is of the order 0.0003 when translated into a shift in $\sin ^{2} \theta_{\mathrm{W}}$.

\section{Acknowledgments}

We would like to thank the MRST collaboration for delivering a modified version of the PDFs of Ref. [10] in which the $\mathcal{O}(\alpha)$ are switched off. Moreover, we gratefully acknowledge V. Lendermann, H. Spiesberger, J. Stirling and A. Tapper for valuable discussions about the treatment of photon radiation off quarks in the analysis of DIS data and its implication for the use of the new MRST PDFs. This work was supported in part by INTAS 03-514007 .

\section{Appendix}

\section{Calculation of photon-induced cross sections}

As explained in Section 5.1 in detail, a consistent evaluation of $\mathcal{O}(\alpha)$ corrections to quark-induced hadronic processes includes an evaluation of related photon-induced channels. In neutrino DIS we have the leading-order processes $\nu_{\mu} q \rightarrow \nu_{\mu} q$ and $\nu_{\mu} q \rightarrow \mu^{-} q^{\prime}$, with $q$ and $q^{\prime}$ generically denoting the relevant quarks and antiquarks. The corresponding photon-induced channels are $\nu_{\mu} \gamma \rightarrow \nu_{\mu} q \bar{q}$ and $\nu_{\mu} \gamma \rightarrow \mu^{-} q^{\prime} \bar{q}$. The matrix elements for these reactions obviously follow upon crossing from the bremsstrahlung processes $\nu_{\mu} q \rightarrow \nu_{\mu} q \gamma$ and $\nu_{\mu} q \rightarrow \mu^{-} q^{\prime} \gamma$, which already entered the calculation of $\mathcal{O}(\alpha)$ corrections described in Ref. [15]. Alternatively they can also be derived upon crossing the results for the real $\mathcal{O}(\alpha)$ corrections to Drell-Yan-like W production as explicitly given in Ref. [39]. We set the coupling factors such that the squared matrix element is proportional to $\alpha^{2} \alpha(0)$, where $\alpha$ is equal to $\alpha(0), \alpha\left(M_{\mathrm{Z}}\right)$, or $\alpha_{G_{\mu}}$ according to the respective IPS; the factor $\alpha(0)$ is taken independent of the IPS, because this is the correct effective coupling for the incoming photon which is on shell. Since the matrix elements have been derived for massless fermions the integration over the domains of collinear initial-state splittings $\gamma \rightarrow f \bar{f}$ requires a restoration of finite-fermion-mass effects. The resulting mass-singular logarithms of initial-state quarks are absorbed into the photon PDF as described in Section 5.1.

In the following we describe three simple methods to restore such effects in a generic reaction $a\left(p_{a}\right)+\gamma(k) \rightarrow f\left(p_{f}\right)+X$, where $a$ stands for any massless incoming parton and $f$ is the light fermion or antifermion whose mass effects we are interested in. The momenta of the particles are given in the parentheses. The remainder $X$ may contain additional light fermions which can be treated in the same way as $f$. The results obtained with the three methods are in mutual numerical agreement, where the dipole subtraction method yields the smallest integration error when using the same statistics in all the methods.

\section{(i) Effective collinear factor}

The collinear singularity in the squared matrix element $\left|\mathcal{M}_{a \gamma \rightarrow f X}\right|^{2}$ occurs if the angle $\theta_{f}$ between $f$ and the incoming $\gamma$ becomes small; in this limit the scalar product $\left(k p_{f}\right)$ 
is of $\mathcal{O}\left(m_{f}^{2}\right)$, where $m_{f}$ is the small mass of $f$. Neglecting terms that vanish in the limit $m_{f} \rightarrow 0$ the polarization-summed squared matrix element (including the average over initial-state polarizations) asymptotically behaves like [40]

$$
\sum_{\mathrm{pol}}\left|\mathcal{M}_{a \gamma \rightarrow f X}\left(p_{a}, k\right)\right|^{2} \underset{k p_{f} \rightarrow 0}{\sim} \frac{Q_{f}^{2} e^{2}}{x\left(k p_{f}\right)}\left[1-2 x(1-x)+\frac{m_{f}^{2}}{k p_{f}} x\right] \sum_{\mathrm{pol}}\left|\mathcal{M}_{a \bar{f} \rightarrow X}\left(p_{a}, p_{\bar{f}}=x k\right)\right|^{2},
$$

where $x=1-p_{f}^{0} / k^{0}, Q_{f} e$ is the electric charge of $f$, and $\mathcal{M}_{a \bar{f} \rightarrow X}$ is the matrix element of the related process which results from $a \gamma(\rightarrow a f \bar{f}) \rightarrow f X$ upon cutting the $\bar{f}$ line if this process proceeds in the collinear limit mainly via the $\gamma \rightarrow f \bar{f}$ splitting. The incoming momenta relevant in the different matrix elements are given in parentheses.

The factorization formula (A.1) can be used to relate the two versions for the squared matrix element $\left|\mathcal{M}_{a \gamma \rightarrow f X}\right|^{2}$ obtained with a finite or vanishing fermion mass $m_{f}$, just by eliminating $\left|\mathcal{M}_{a \bar{f} \rightarrow X}\right|^{2}$. The result is

$$
\left.\sum_{\mathrm{pol}}\left|\mathcal{M}_{a \gamma \rightarrow f X}\left(p_{a}, k\right)\right|^{2} \underset{k p_{f} \rightarrow 0}{\widetilde{T}} g\left(m_{f}, x, k^{0}, \theta_{f}\right) \sum_{\mathrm{pol}}\left|\mathcal{M}_{a \gamma \rightarrow f X}\left(p_{a}, k\right)\right|^{2}\right|_{m_{f}=0}
$$

with the "effective collinear factor"

$$
\begin{aligned}
& g\left(m_{f}, x, k^{0}, \theta_{f}\right) \\
& =\frac{(1-x)^{2}}{(1-x)^{2}+x^{2}} \frac{4\left(k^{0}\right)^{2} \sin ^{2} \frac{\theta_{f}}{2}\left[m_{f}^{2}+4\left(k^{0}\right)^{2} \sin ^{2} \frac{\theta_{f}}{2}(1-x)^{2}\left((1-x)^{2}+x^{2}\right)\right]}{\left[m_{f}^{2}+4\left(k^{0}\right)^{2} \sin ^{2} \frac{\theta_{f}}{2}(1-x)^{2}\right]^{2}} .
\end{aligned}
$$

The squared matrix element on the 1.h.s. of Eq. (A.2) can be integrated over the phase space for a massless $f$, yielding the correct result for a massive $f$ up to terms that are suppressed by powers of $m_{f}$. This means that the collinear singularity is regularized by the correct cutoff term $\ln m_{f}$ supplemented with the correct constant contributions for $m_{f} \rightarrow 0$. However, there are spurios terms of $\mathcal{O}\left(m_{f} / Q\right)$ where $Q$ is a typical scale in the process.

\section{(ii) Phase-space slicing}

In the collinear limit $\theta_{f} \rightarrow 0, m_{f} \rightarrow 0$ the phase-space volume element $\mathrm{d} \Phi_{a \gamma \rightarrow f X}\left(p_{a}, k\right)$ can be factorized as

$$
\int_{\theta_{f}<\Delta \theta} \mathrm{d} \Phi_{a \gamma \rightarrow f X}\left(p_{a}, k\right) \underset{\Delta \sigma \rightarrow 0}{\widetilde{\theta \rightarrow}} \int_{0}^{1} \mathrm{~d} x \int \mathrm{d} \Phi_{a \bar{f} \rightarrow X}\left(p_{a}, x k\right) \frac{(1-x)\left(k^{0}\right)^{2}}{2(2 \pi)^{2}} \int_{0}^{\Delta \theta} \mathrm{d} \theta_{f} \sin \theta_{f},
$$

where $\Delta \theta \ll 1$ is a small cutoff parameter defining the collinear region. Here we also used that the incoming parton $a$ is massless. Making use of this factorized phase space and of the asymptotic form (A.1) of the squared matrix element, the integral over small emission angles $\theta_{f}$ can be carried out, yielding

$$
\left.\sigma_{a \gamma \rightarrow f X}\left(p_{a}, k\right)\right|_{\theta_{f}<\Delta \theta}=\frac{1}{4 p_{a} k} \int_{\theta_{f}<\Delta \theta} \mathrm{d} \Phi_{a \gamma \rightarrow f X}\left(p_{a}, k\right) \sum_{\mathrm{pol}}\left|\mathcal{M}_{a \gamma \rightarrow f X}\left(p_{a}, k\right)\right|^{2}
$$




$$
\begin{aligned}
& =\frac{Q_{f}^{2} \alpha}{2 \pi} \int_{0}^{1} \mathrm{~d} x H\left(m_{f}, x, k^{0}, \Delta \theta\right) \frac{1}{4\left(p_{a} k\right) x} \int \mathrm{d} \Phi_{a \bar{f} \rightarrow X}\left(p_{a}, x k\right) \\
& \quad \times \sum_{\mathrm{pol}}\left|\mathcal{M}_{a \bar{f} \rightarrow X}\left(p_{a}, p_{\bar{f}}=x k\right)\right|^{2}+\mathcal{O}(\Delta \theta), \\
& =N_{\mathrm{c}, f} \frac{Q_{f}^{2} \alpha}{2 \pi} \int_{0}^{1} \mathrm{~d} x H\left(m_{f}, x, k^{0}, \Delta \theta\right) \sigma_{a \bar{f} \rightarrow X}\left(p_{a}, p_{\bar{f}}=x k\right)+\mathcal{O}(\Delta \theta)
\end{aligned}
$$

with the auxiliary function

$$
H\left(m_{f}, x, k^{0}, \Delta \theta\right)=P_{f \gamma}(x) \ln \left(\frac{\Delta \theta^{2}\left(k^{0}\right)^{2}(1-x)^{2}}{m_{f}^{2}}\right)+2 x(1-x)
$$

containing the collinear singularity. The splitting function $P_{f \gamma}(x)$ is defined in Eq. (5.2), and $N_{\mathrm{c}, f}$ is the colour factor for the fermion $f\left(N_{\mathrm{c} \text {,lepton }}=1, N_{\mathrm{c}, \text { quark }}=3\right)$.

The remaining phase-space integration over the non-collinear region $\theta_{f}>\Delta \theta$ can be carried out for a massless $f$ (provided no other singularities are connected with $f$ ) without modifications.

\section{(iii) Dipole subtraction}

The general idea of a subtraction method is to subtract and to add a simple auxiliary function from the singular integrand. This auxiliary function has to be chosen such that it cancels all singularities of the original integrand so that the phase-space integration of the difference can be performed numerically. Moreover, the auxiliary function has to be simple enough so that it can be integrated over the singular regions analytically, when the subtracted contribution is added again. The dipole subtraction method, originally worked out for massless QCD [33], provides a general algorithm for the construction of this auxiliary function and for its integrated counterpart.

The dipole subtraction method for photon radiation off massive and massless fermions has been described in Ref. [34] and Ref. [35]. Following the same strategy, we work out a similar formalism for the collinear splitting $\gamma \rightarrow f \bar{f}$ of a photon in the initial state into a light $f \bar{f}$ pair. More generalizations of the dipole formalism of Ref. [34] will be published elsewhere [37].

The function that is subtracted from the integrand $\sum_{\mathrm{pol}}\left|\mathcal{M}_{a \gamma \rightarrow f X}\left(p_{a}, k\right)\right|^{2}$, which is derived for $m_{f}=0$, is defined as follows, ${ }^{2}$

$$
\left|\mathcal{M}_{\mathrm{sub}}\right|^{2}=Q_{f}^{2} e^{2} h_{\gamma a}\left(k, p_{f}, p_{a}\right) \sum_{\mathrm{pol}}\left|\mathcal{M}_{a \bar{f} \rightarrow X}\left(p_{a}, p_{\bar{f}}=x_{\gamma a} k, \tilde{k}_{X}\right)\right|^{2}
$$

with the radiator function

$$
h_{\gamma a}\left(k, p_{f}, p_{a}\right)=\frac{P_{f \gamma}\left(x_{\gamma a}\right)}{x_{\gamma a}\left(p_{f} k\right)}, \quad x_{\gamma a}=\frac{p_{a} k-p_{f} k-p_{a} p_{f}}{p_{a} k} .
$$

\footnotetext{
${ }^{2}$ This construction closely follows the case of an "initial-state emitter" and of an "initial-state spectator" in the case of photon or gluon radiation, as described in Refs. [33-35].
} 
The final-state momenta entering the squared matrix element on the r.h.s. of Eq. (A.7), generically denoted $\tilde{k}_{X}$, follow from the original momenta $k_{X}$ upon the Lorentz transformation

$$
\tilde{k}_{X}^{\mu}=\Lambda^{\mu}{ }_{\nu} k_{X}^{\nu}
$$

with

$$
\Lambda_{\nu}^{\mu}=g^{\mu}{ }_{\nu}-\frac{(P+\tilde{P})^{\mu}(P+\tilde{P})_{\nu}}{P^{2}+P \tilde{P}}+\frac{2 \tilde{P}^{\mu} P_{\nu}}{P^{2}}, \quad P^{\mu}=p_{a}^{\mu}+k^{\mu}-p_{f}^{\mu}, \quad \tilde{P}^{\mu}=p_{a}^{\mu}+x_{\gamma a} k^{\mu} .
$$

It is straightforward to check that $\left|\mathcal{M}_{\text {sub }}\right|^{2}$ possesses the same asymptotic behaviour as $\sum_{\text {pol }}\left|\mathcal{M}_{a \gamma \rightarrow f X}\right|^{2}$ in Eq. (A.1) for $m_{f}=0$. Thus, the difference $\sum_{\text {pol }}\left|\mathcal{M}_{a \gamma \rightarrow f X}\right|^{2}-\left|\mathcal{M}_{\text {sub }}\right|^{2}$ can be integrated numerically for $m_{f}=0$. The correct dependence of $\left|\mathcal{M}_{\text {sub }}\right|^{2}$ (and the related kinematics) on a finite $m_{f}$ is, however, needed when this function is integrated over $\theta_{f}$ leading to the collinear singularity for $\theta_{f} \rightarrow 0$. The details of this part of the calculation will be described elsewhere [37]; here we give only the final result:

$$
\sigma_{a \gamma \rightarrow f X}^{\mathrm{sub}}\left(p_{a}, k\right)=N_{\mathrm{c}, f} \frac{Q_{f}^{2} \alpha}{2 \pi} \int_{0}^{1} \mathrm{~d} x \mathcal{H}\left(m_{f}, x, p_{a} k\right) \sigma_{a \bar{f} \rightarrow X}\left(p_{a}, x k\right)
$$

with the auxiliary function

$$
\mathcal{H}\left(m_{f}, x, p_{a} k\right)=P_{f \gamma}(x) \ln \left(\frac{2 p_{a} k(1-x)^{2}}{m_{f}^{2}}\right)+2 x(1-x) .
$$

The contribution $\sigma_{a \gamma \rightarrow f X}^{\mathrm{sub}}$ simply has to be added to the result for the cross section obtained from the integral of the difference $\sum_{\mathrm{pol}}\left|\mathcal{M}_{a \gamma \rightarrow f X}\right|^{2}-\left|\mathcal{M}_{\text {sub }}\right|^{2}$.

\section{References}

[1] G. P. Zeller et al. [NuTeV Collaboration], Phys. Rev. Lett. 88 (2002) 091802 [Erratum-ibid. 90 (2003) 239902] [hep-ex/0110059].

[2] The LEP Collaborations, the LEP Electroweak Working Group, and SLD Electroweak Group and SLD Heavy Flavour Group, hep-ex/0412015.

[3] S. Davidson, S. Forte, P. Gambino, N. Rius and A. Strumia, JHEP 0202 (2002) 037 [hep-ph/0112302].

[4] R. H. Bernstein [NuTeV Collaboration], J. Phys. G 29 (2003) 1919 [hep-ex/0210061].

[5] P. Gambino, hep-ph/0211009.

[6] C. Giunti and M. Laveder, hep-ph/0202152;

W. Loinaz, N. Okamura, T. Takeuchi and L. C. R. Wijewardhana, Phys. Rev. D 67 (2003) 073012 [hep-ph/0210193];

A. Kurylov, M. J. Ramsey-Musolf and S. Su, Nucl. Phys. B 667 (2003) 321 [hep$\mathrm{ph} / 0301208]$;

W. Loinaz, N. Okamura, S. Rayyan, T. Takeuchi and L. C. R. Wijewardhana, Phys. 
Rev. D 70 (2004) 113004 [hep-ph/0403306];

O. Brein, B. Koch and W. Hollik, hep-ph/0408331;

T. Takeuchi and W. Loinaz, hep-ph/0410201;

J. S. Ma, J. M. Conrad, M. Sorel and G. P. Zeller, hep-ex/0501011.

[7] J. T. Londergan, Nucl. Phys. Proc. Suppl. 141 (2005) 68 [hep-ph/0408243].

[8] G. P. Zeller et al. [NuTeV Collaboration], Phys. Rev. D 65 (2002) 111103 [Erratumibid. D 67 (2003) 119902] [hep-ex/0203004];

S. Kretzer, F. Olness, J. Pumplin, D. Stump, W. K. Tung and M. H. Reno, Phys. Rev. Lett. 93 (2004) 041802 [hep-ph/0312322];

J. Alwall and G. Ingelman, Phys. Rev. D 70 (2004) 111505 [hep-ph/0407364];

Y. Ding, R. G. Xu and B. Q. Ma, Phys. Lett. B 607 (2005) 101 [hep-ph/0408292] and Phys. Rev. D 71 (2005) 094014 [hep-ph/0505153];

M. Wakamatsu, Phys. Rev. D 71 (2005) 057504 [hep-ph/0411203];

M. Glück, P. Jimenez-Delgado and E. Reya, hep-ph/0501169 and Phys. Rev. Lett. 95 (2005) 022002 [hep-ph/0503103].

[9] K. S. McFarland and S. O. Moch, hep-ph/0306052.

[10] A. D. Martin, R. G. Roberts, W. J. Stirling and R. S. Thorne, Eur. Phys. J. C 39 (2005) 155 [hep-ph/0411040].

[11] G. A. Miller and A. W. Thomas, Int. J. Mod. Phys. A 20 (2005) 95 [hep-ex/0204007];

G. P. Zeller et al. [NuTeV Collaboration], hep-ex/0207052;

S. Kovalenko, I. Schmidt and J. J. Yang, Phys. Lett. B 546 (2002) 68 [hep$\mathrm{ph} / 0207158]$

S. A. Kulagin, Phys. Rev. D 67 (2003) 091301 [hep-ph/0301045]; hep-ph/0406220 and Nucl. Phys. Proc. Suppl. 139 (2005) 213 [hep-ph/0409057];

S. J. Brodsky, I. Schmidt and J. J. Yang, Phys. Rev. D 70 (2004) 116003 [hep$\mathrm{ph} / 0409279]$

M. Hirai, S. Kumano and T. H. Nagai, Phys. Rev. D 71 (2005) 113007 [hep$\mathrm{ph} / 0412284]$

S. A. Kulagin and R. Petti, hep-ph/0412425.

[12] S. Kretzer and M. H. Reno, hep-ph/0307023;

B. A. Dobrescu and R. K. Ellis, hep-ph/0310154.

[13] W. J. Marciano and A. Sirlin, Phys. Rev. D 22 (1980) 2695 [Erratum-ibid. D 31 (1985) 213] and Nucl. Phys. B 189 (1981) 442.

[14] D. Y. Bardin and V. A. Dokuchaeva, On The Radiative Corrections To The Neutrino Deep Inelastic Scattering, JINR-E2-86-260.

[15] K. P. O. Diener, S. Dittmaier and W. Hollik, Phys. Rev. D 69 (2004) 073005 [hep$\mathrm{ph} / 0310364]$.

[16] A. B. Arbuzov, D. Y. Bardin and L. V. Kalinovskaya, hep-ph/0407203. 
[17] R. Petti [NOMAD Collaboration], hep-ex/0411032.

[18] C. H. Llewellyn Smith, Nucl. Phys. B 228 (1983) 205.

[19] E. A. Paschos and L. Wolfenstein, Phys. Rev. D 7 (1973) 91.

[20] A. Denner, Fortsch. Phys. 41 (1993) 307.

[21] M. Böhm, H. Spiesberger and W. Hollik, Fortsch. Phys. 34 (1986) 687;

W. F. Hollik, Fortsch. Phys. 38 (1990) 165.

[22] A. Sirlin, Phys. Rev. D 22 (1980) 971.

[23] M. Consoli, W. Hollik and F. Jegerlehner, CERN-TH-5527-89, Presented at Workshop on Z Physics at LEP and Phys. Lett. B 227 (1989) 167.

[24] J. Fleischer, O. V. Tarasov and F. Jegerlehner, Phys. Lett. B 319 (1993) 249.

[25] T. Kinoshita, J. Math. Phys. 3 (1962) 650;

T. D. Lee and M. Nauenberg, Phys. Rev. 133 (1964) B1549.

[26] E. A. Kuraev and V. S. Fadin, Sov. J. Nucl. Phys. 41 (1985) 466 [Yad. Fiz. 41 (1985) 733];

G. Altarelli and G. Martinelli, in Physics at LEP, eds. J. Ellis and R. Peccei, (CERN 86-02, Geneva, 1986), Vol. 1, p. 47;

O. Nicrosini and L. Trentadue, Phys. Lett. B 196 (1987) 551 and Z. Phys. C 39 (1988) 479;

F. A. Berends, W. L. van Neerven and G. J. H. Burgers, Nucl. Phys. B 297 (1988) 429 [Erratum-ibid. B 304 (1988) 921];

A. B. Arbuzov, Phys. Lett. B 470 (1999) 252 [hep-ph/9908361].

[27] B. Humpert and W. L. van Neerven, Nucl. Phys. B 184 (1981) 225.

[28] J. Kripfganz and H. Perlt, Z. Phys. C 41 (1988) 319;

H. Spiesberger, Phys. Rev. D 52 (1995) 4936 [hep-ph/9412286];

M. Roth and S. Weinzierl, Phys. Lett. B 590 (2004) 190 [hep-ph/0403200].

[29] V. Lendermann, H. Spiesberger, J. Stirling and A. Tapper, private communications.

[30] S. Eidelman et al. [Particle Data Group Collaboration], Phys. Lett. B 592 (2004) 1.

[31] P. Azzi et al. [CDF and D0 Collaborations, and Tevatron Electroweak Working Group], hep-ex/0404010.

[32] F. Jegerlehner, DESY 01-029, LC-TH-2001-035 [hep-ph/0105283].

[33] S. Catani and M. H. Seymour, Phys. Lett. B 378 (1996) 287 [hep-ph/9602277] and Nucl. Phys. B 485 (1997) 291 [Erratum-ibid. B 510 (1997) 291] [hep-ph/9605323].

[34] S. Dittmaier, Nucl. Phys. B 565 (2000) 69 [hep-ph/9904440]. 
[35] M. Roth, PhD thesis, ETH Zürich No. 13363 (1999), hep-ph/0008033.

[36] A. Bredenstein, S. Dittmaier and M. Roth, hep-ph/0506005, to appear in Eur. Phys. J. C.

[37] S. Dittmaier, in preparation.

[38] M. Faisst, J. H. Kuhn, T. Seidensticker and O. Veretin, Nucl. Phys. B 665 (2003) 649 [hep-ph/0302275].

[39] S. Dittmaier and M. Krämer, Phys. Rev. D 65 (2002) 073007 [hep-ph/0109062].

[40] S. Catani, S. Dittmaier, M. H. Seymour and Z. Trocsanyi, Nucl. Phys. B 627 (2002) 189 [hep-ph/0201036]. 